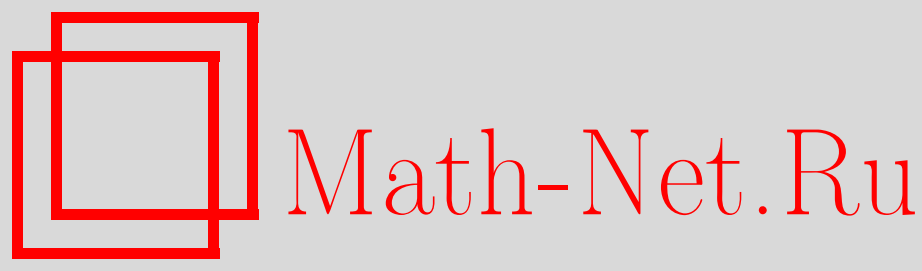

А. М. Семихатов, Факторизуемые ленточные квантовые группы в логарифмических конформных теориях поля, TMФ, 2008, том 154, номер 3, 510-535

DOI: https://doi.org/10.4213/tmf6184

Использование Общероссийского математического портала Math-Net.Ru подразумевает, что вы прочитали и согласны с пользовательским соглашением http://www . mathnet.ru/rus/agreement

Параметры загрузки:

IP : 54.80 .97 .219

26 апреля 2023 г., $11: 33: 13$

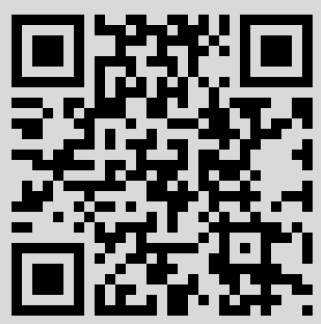




\title{
ФИЗИКА
}

Том 154, № 3

март, 2008

2008 г.

А. М. Семихатов*

\section{ФАКТОРИЗУЕМЫЕ ЛЕНТОЧНЫЕ КВАНТОВЫЕ ГРУППЫ В ЛОГАРИФМИЧЕСКИХ КОНФОРМНЫХ ТЕОРИЯХ ПОЛЯ}

\begin{abstract}
Дан обзор свойств квантовых групп, возникающих как двойственные по Каждану-Люстигу логарифмическим моделям конформной теории поля. Эти квантовые группы в четных корнях из единицы не являются квазитреугольными, но оказываются факторизуемыми и обладают ленточной структурой; представление модулярной группы на их центре совпадает с представлением на обобщенных характерах киральной алгебры в моделях логарифмических конформных теорий поля.
\end{abstract}

Ключевые слова: квантовые группы, факторизуемая структура, ленточная структура, модулярная группа, кольцо Гротендика, соответствие Каждана-Люстига, логарифмические конформные теории поля.

\section{1. ВВЕДЕНИЕ}

Связь между квантовыми группами и конформной теорией поля, которая обсуждалась начиная с работ [1]-[4], была сформулирована в контексте вертекс-операторных алгебр как соответствие Каждана-Люстига [5]. В очень широком смысле (и очень приблизительно) соответствие Каждана-Люстига утверждает, что всякий раз, когда "нечто происходит" в категории представлений вертекс-операторной алгебры, "нечто похожее происходит" в категории представлений подходящей квантовой группы; другими словами, имеется функтор, связывающий эти две категории, хотя он и не обязан быть точным ни слева, ни справа. В этом широком смысле соответствие Каждана-Люстига является скорее принципом, чем точным утверждением; подробности, связанные с функтором, подлежат выяснению в каждом конкретном случае. Для рациональных конформных теорий поля некоторое усложнение возникает вследствие того, что выбранная категория представлений вертекс-операторной алгебры является полупростой, а квантово-групповая категория представлений, как правило, не является, а потому дополнительное "полуупрощение" (взятие фактора по tilting-модулям) оказывается необходимым для обеспечения эквивалентности [6]. Но в логарифмических конформных теориях поля [7], [8] категория представлений

*Физический Институт им. П. Н. Лебедева РАН, Москва, Россия.

E-mail: ams@sci.lebedev.ru 
неполупроста уже в конформной теории, а потому априори не требуется взятие фактора и в терминах квантовой группы. Соответствие Каждана-Люстига, продолженное на логарифмическую область, демонстрирует замечательные свойства, в частности, расширение до представлений модулярной группы [9]-[11].

Более "физический" взгляд на соответствие Каждана-Люстига исходит из наблюдения, что экранирующие операторы (скрининги), коммутирующие с данной вертекс-операторной алгеброй, порождают квантовую группу, и, более того, данная вертекс-операторная алгебра и данная квантовая группа характеризуются тем, что являются коммутантами друг друга,

[вертекс-операторная алгебра, квантовая группа] $=0$,

так что каждый из объектов в этом соотношении позволяет реконструировать другой. Но и эта картина применима скорее как принцип, нежели как точное утверждение, а потому также требует прояснения. Во-первых, собственно скрининги порождают только верхнетреугольную подалгебру в данной квантовой группе; полную квантовую группу надо восстанавливать, или вводя операторы удаления контура (см. [12] и приведенную там библиографию), или, несколько более формально, путем взятия дубля Дринфельда [9], [13]. Во-вторых, при поиске коммутанта квантовой группы необходимо указать, где он ищется, т.е. какие свободно-полевые операторы рассматриваются (в частности, каковы допустимые "импульсы" вертексных операторов и допускаются ли вообще вертексные операторы; ср., например, с работами [14], [15] в нелогарифмическом случае).

Для нескольких логарифмических конформных теорий поля оказалось, что соответствие Каждана-Люстига обладает очень хорошими свойствами [9], [10], [13], [11], демонстрируя "улучшение" по сравнению с рациональным случаем. Несколько эвристически подобное "улучшение" можно связать с тем, что состав полей в логарифмических моделях определяется не когомологиями, а ядром скрининга(ов) (точнее, ядром дифференциала, построенного из скринингов; при этом рациональные модели даются в точности когомологиями такого дифференциала, ср. с [16], [17]). Наиболее замечательно, что соответствие Каждана-Люстига распространяется на представления модулярной группы. Напомним, что представление модулярной группы в логарифмической конформно-полевой модели порождается из характеров $\chi_{a}(\tau)$ модели $\mathcal{T}$ - и $\mathcal{S}$-преобразованиями, причем $\mathcal{S}$-преобразованные характеры выражаются формулой

$$
\chi_{a}\left(-\frac{1}{\tau}\right)=\sum_{b} S_{a b} \chi_{b}(\tau)+\sum_{b^{\prime}} S_{a b^{\prime}}^{\prime} \psi_{b^{\prime}}(\tau),
$$

в которую входят некоторые функции $\psi_{b^{\prime}}$, не являющиеся характерами [18]-[20], [13], причем

$$
\psi_{a^{\prime}}\left(-\frac{1}{\tau}\right)=\sum_{b} S_{a^{\prime} b}^{\prime} \chi_{b}(\tau)+\sum_{b^{\prime}} S_{a^{\prime} b^{\prime}}^{\prime} \psi_{b^{\prime}}(\tau)
$$

(взятые вместе, $\chi$ и $\psi$ можно называть обобщенными или расширенными характерами за неимением лучшего названия). С другой стороны, общую теорию, разработанную в совершенно ином контексте для квантовых групп в [21] (см. также [22], [23]), 
можно применить к квантовым группам, которые являются двойственными логарифмическим конформным теориям поля, и в результате представление модулярной группы удается определить на центре квантовой группы. Это представление оказывается эквивалентным тому, которое порождается из характеров.

Другой аспект, в котором логарифмические конформные теории поля и соответствующие (“двойственные”) квантовые группы демонстрируют подобие, - это алгебры Верлинде (алгебры слияния) и кольцо Гротендика. Существующие результаты подсказывают, что кольцо Гротендика двойственной по Каждану-Люстигу квантовой группы совпадает или "очень тесно связано" с алгеброй слияний для представлений киральной алгебры в соответствующей конформной теории поля. Здесь уместны два замечания. Во-первых, сравнение кольца Гротендика с алгеброй слияний подразумевает, что последняя понимается в " $K_{0}$-варианте", когда все неразложимые представления "насильно" заменены прямыми суммами (ср. с обсуждением этого вопроса в [19] $)^{1)}$. Во-вторых, когда логарифмическая конформная теория поля имеет рациональную подтеорию, представления этой рациональной подтеории исключаются из сравнения (это, впрочем, достаточно естественно, ср. с [26]).

Квантовые группы, до сих пор возникавшие в качестве двойственных логарифмическим конформным теориям поля, - это квантовая $s \ell(2)$ и несколько более сложная квантовая группа (являющаяся фактором произведения двух квантовых $s \ell(2))$. Они двойственны логарифмическим конформным теориям поля из классов $(p, 1)$ и $\left(p, p^{\prime}\right)$-моделей соответственно. В обоих случаях параметр $q$ в двойственной по Каждану-Люстигу квантовой группе равен четному корню из единицы, а квантовая группа обладает рядом характерных свойств, которые можно гипотетически считать общими для квантовых групп, двойственных логарифмическим конформным моделям. Эти свойства и те структуры, на которых они основаны, и рассматриваются в данном обзоре. В настоящий момент имеется лишь вывод этих свойств в весьма "приземленных" терминах - прямым вычислением, которое до известной степени затемняет общую картину. Ниже мы опускаем вычислительные подробности и фокусируем внимание на окончательных результатах и на взаимодействии различных структур, связанных с квантовой группой.

Мы строим изложение в соответствии с тем, что видится со стороны квантовой группы, следуя при этом идеологии и результатам работ [9], [10], [13], [11]. Что касается необходимых экскурсов в логарифмические конформные теории поля (см. [8], [27], [25], [28], [19], [9], [13] и приведенную там библиографию), мы ограничимся необходимым для обсуждения схожих черт с квантово-групповыми структурами. Когда нам нужно быть конкретными (а это почти всегда так, поскольку мы не утверждаем здесь никакой особенной общности), мы выбираем простейший из двух основных примеров, квантовую группу $\overline{\mathcal{U}}_{\mathfrak{q}} s \ell(2)$, двойственную логарифмическим $(p, 1)$-моделям конформной теории поля, но везде где можно мы указываем

\footnotetext{
1) При наличии неразложимых представлений, разумеется, можно (и даже более интересно) рассматривать алгебры слияния, в которых неразложимые представления учитываются "честным" образом, т.е. не заменяются прямой суммой своих неприводимых подфакторов [24], [25]. Соответствие с квантовыми группами может распространиться с " $K_{0} /$ Гротендик-подобных" слияний и на этот случай (см. также п. 3.3 .2 ниже).
} 
свойства, присущие также квантовой группе $\mathcal{U}_{p, p^{\prime}}$, двойственной логарифмическим $\left(p, p^{\prime}\right)$-моделям.

Квантовой группой, двойственной логарифмическим $(p, 1)$-моделям, является $\overline{\mathcal{U}}_{\mathfrak{q}} s \ell(2)$ с параметром, равным четному корню из единицы:

$$
\mathfrak{q}=e^{\frac{i \pi}{p}}
$$

Три ее генератора $E, F$ и $K$ удовлетворяют соотношениям

$$
K E K^{-1}=\mathfrak{q}^{2} E, \quad K F K^{-1}=\mathfrak{q}^{-2} F, \quad[E, F]=\frac{K-K^{-1}}{\mathfrak{q}-\mathfrak{q}^{-1}}
$$

и "связям"

$$
E^{p}=F^{p}=0, \quad K^{2 p}=1 .
$$

Заметим, что из равенств $(1.3),(1.4)$ уже следует, что $E^{p}, F^{p}$ и $K^{2 p}$ являются центральными, и это позволяет наложить условия (1.5) (но элемент $K^{p}$, также являющийся центральным, не полагается равным единице, что и составляет отличие от более популярного варианта так называемой малой квантовой $s \ell(2))$. В результате группа $\overline{\mathcal{U}}_{\mathfrak{q}} s \ell(2)$ оказывается $2 p^{3}$-мерной. Квантовая группа $\mathcal{U}_{p, p^{\prime}}$, двойственная логарифмическим $\left(p, p^{\prime}\right)$-моделям, $2 p^{3} p^{\prime 3}$-мерна. Заметим, что “связь” для ее картановского генератора имеет вид $K^{2 p p^{\prime}}=1$.

Структура алгебры Хопфа для $\overline{\mathcal{U}}_{\mathfrak{q}} s \ell(2)$ (коумножение $\Delta$, коединица $\epsilon$ и антипод $S$ ) описывается как

$$
\begin{gathered}
\Delta(E)=1 \otimes E+E \otimes K, \quad \Delta(F)=K^{-1} \otimes F+F \otimes 1, \quad \Delta(K)=K \otimes K, \\
\epsilon(E)=\epsilon(F)=0, \quad \epsilon(K)=1, \\
S(E)=-E K^{-1}, \quad S(F)=-K F, \quad S(K)=K^{-1} .
\end{gathered}
$$

Простота соотношений (1.4)-(1.6) несколько обманчива. Данная квантовая группа $\left(\right.$ как и $\left.\mathcal{U}_{p, p^{\prime}}\right)$ обладает интересными алгебраическими свойствами, причем центральную роль играет ее центр.

Центр квантовой группы и структуры на нем. В терминах квантовых групп основной ареной соответствия Каждана-Люстига является центр Z квантовой группы. Он, конечно, содержит ("квантовые") элементы Казимира и порожденную ими алгебру, но этим не исчерпывается.

Центр несет представление группы $S L(2, \mathbb{Z})$, для определения которого требуются три типа структур (см. [21]-[23]): отображения Дринфельда и Радфорда $\chi$ и $\widehat{\phi}$, а также ленточный элемент $\boldsymbol{v}$. Действие элемента $\mathcal{S}=\left(\begin{array}{cc}0 & 1 \\ -1 & 0\end{array}\right) \in S L(2, \mathbb{Z})$ на центре определяется из диаграммы

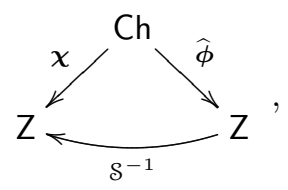

где $\mathrm{Ch}$ - пространство $q$-характеров (линейных функционалов, инвариантных относительно коприсоединенного действия), а действие $\mathcal{T}=\left(\begin{array}{ll}1 & 1 \\ 0 & 1\end{array}\right) \in S L(2, \mathbb{Z})$ по сути

5 Теоретическая и математическая физика, т. 154, № 3, 2008 г. 
определяется ленточным элементом (умножением на него):

$$
\mathrm{Z} \stackrel{v}{\longrightarrow} \mathrm{Z}
$$

(в нашем определении по сравнению со стандартными соглашениями $\widehat{\phi}$ переставлено со своим обратным.)

Можно посмотреть на центр следующим образом: сначала выделить некоторое число центральных элементов, связанных со следами по неприводимым представлениям, а далее ввести подходящие псевдоследы. След (“квантовый” след) по неприводимому представлению дает элемент из $\mathrm{Ch}$ - функционал на квантовой группе, инвариантный относительно коприсоединенного представления; эти инвариантные функционалы ( $q$-характеры) можно далее отобразить в центральные элементы одним из отображений в (1.7). Таким образом не покрывается весь центр, но проективные модули над квантовой группой дают дополнительные $q$-характеры, получаемые взятием следов, неформально говоря, от недиагональных (в терминах фильтрации проективных модулей) компонент квантово-группового действия. Это дает базис $\gamma_{A}$ в пространстве Ch $q$-характеров, а поэтому и базис в центре.

Отображение Дринфельда $\chi: \mathrm{Ch} \rightarrow \mathrm{Z}$ является также изоморфизмом ассоциативных коммутативных алгебр. Поэтому центр содержит кольцо Гротендика квантовой группы (его изоморфный образ), которое таким образом вкладывается в бо́льшую ассоциативную коммутативную алгебру ${ }^{2}$.

В разделе 2 рассматривается отображение Радфорда $\widehat{\phi}$. В разделе 3 перечисляются необходимые факты о (неприводимых и проективных) представлениях соответствующих квантовых групп, а также обсуждаются их кольца Гротендика. В разделе 4 рассматриваются "матрица" $M$ (монодромии), отображение Дринфельда и ленточный элемент. Вместе с отображением Радфорда они участвуют в определении действия модулярной группы, которое дается в разделе 5.

\section{2. ОТОБРАЖЕНИЕ РАДФОРДА И СВЯЗАННЫЕ С НИМ СТРУКТУРЫ}

Мы рассматриваем отображение Радфорда $\widehat{\phi}: U^{*} \rightarrow U$; конструкция для $\widehat{\phi}$ и его обратного использует коинтеграл и интеграл.

\section{1. Интеграл и коинтеграл.}

2.1.1. Интеграл. Для алгебры Хопфа $U$ правъй интеграл $\boldsymbol{\lambda}$ является линейным функционалом на $U$ таким, что

$$
(\boldsymbol{\lambda} \otimes \mathrm{id}) \Delta(x)=\boldsymbol{\lambda}(x) 1 \quad \forall x \in U
$$

В конечномерной алгебре Хопфа такой функционал существует и единствен с точностью до множителя [30].

${ }^{2)}$ Тот факт, что центр содержит образ кольца Гротендика, но больше его, имеет аналог в логарифмических конформных теориях, где действие модулярной группы приводит к расширению набора характеров $\chi_{a}$ киральной алгебры за счет некоторых других функций $\psi_{a^{\prime}}$ [18], [19], [13]; таким образом, как можно полагать, порождается пространство амплитуд на торе (см. также [20]). 
2.1.2. Замечание. Название "интеграл" для такого $\boldsymbol{\lambda} \in U^{*}$ связано с тем, что формула (2.1) выражает также свойство правоинвариантного интегрирования функций на группе. Действительно, если $f$ - функция на группе $G$, то $\Delta(f)$ - такая функция на $G \times G$, что $\Delta(f)(x, y)=f(x y), x, y \in G$. Тогда свойство инвариантности $\int f(? y)=\int f(?)$ можно записать в виде $\left(\int \otimes \mathrm{id}\right) \Delta(f)=\int f$.

2.1.3. Коинтеграл. Двойственный к $\boldsymbol{\lambda}$ объект, интеграл для $U^{*}$, иногда называют коинтегралом для $U$. Приведем его в случае, который только и понадобится в дальнейшем, когда это двусторонний коинтеграл ${ }^{3)} \boldsymbol{\Lambda}$, т.е. такой элемент $U$, что $x \boldsymbol{\Lambda}=\boldsymbol{\Lambda} x=\epsilon(x) \boldsymbol{\Lambda}$ для любого $x \in U$.

Очевидно, коинтеграл определяет вложение тривиального представления $U$ в регулярное представление. Обычно предполагается, что имеет место нормировка $\boldsymbol{\lambda}(\boldsymbol{\Lambda})=1$.

2.2. Отображение Радфорда. Пусть $U$ - алгебра Хопфа с правым интегралом $\boldsymbol{\lambda}$ и двусторонним коинтегралом $\boldsymbol{\Lambda}$. Отображение Радфорда $\widehat{\phi}: U^{*} \rightarrow U$ и его обратное $\widehat{\phi}^{-1}: U \rightarrow U^{*}$ определяются как ${ }^{4)}$

$$
\widehat{\phi}(\beta)=\beta\left(\Lambda^{\prime}\right) \Lambda^{\prime \prime}, \quad \widehat{\phi}^{-1}(x)=\lambda(S(x) ?) .
$$

2.2.1. Лемма [30], [31]. Отображения $\widehat{\phi} и \widehat{\phi}^{-1}$ взаимно обратны и сплетают левые действия алгебры $U$ на $U$ и $U^{*}$; аналогичное утверждение имеет место для правых действий.

Здесь структура левого $U$-модуля на $U^{*}$ задается как $a \neg \beta=\beta(S(a)$ ?), а на $U$ посредством регулярного действия. В частности, при ограничении на пространство $q$-характеров (см. п. П.1) получаем отображение $\widehat{\phi}: C h \rightarrow$ Z. Для полноты рассуждений мы приведем доказательство этой известной леммы.

ДокАзАтЕЛьство. Сначала установим следующее свойство инвариантности интеграла:

$$
\boldsymbol{\lambda}\left(x y^{\prime}\right) y^{\prime \prime}=\boldsymbol{\lambda}\left(x^{\prime} y\right) S^{-1}\left(x^{\prime \prime}\right) .
$$

Действительно ${ }^{5)}, \boldsymbol{\lambda}\left(x y^{\prime}\right) y^{\prime \prime}=\boldsymbol{\lambda}\left(x^{\prime} y^{\prime}\right) S^{-1}\left(x^{\prime \prime \prime}\right) x^{\prime \prime} y^{\prime \prime}=\boldsymbol{\lambda}\left(\left(x^{\prime} y\right)^{\prime}\right) S^{-1}\left(x^{\prime \prime}\right)\left(x^{\prime} y\right)^{\prime \prime}=$ $\boldsymbol{\lambda}\left(x^{\prime} y\right) S^{-1}\left(x^{\prime \prime}\right)$. Отсюда получаем $\widehat{\phi}\left(\widehat{\phi}^{-1}(x)\right)=\widehat{\phi}(\boldsymbol{\lambda}(S(x) ?))=\boldsymbol{\lambda}\left(S(x) \boldsymbol{\Lambda}^{\prime}\right) \boldsymbol{\Lambda}^{\prime \prime}=$ $\boldsymbol{\lambda}\left(S(x)^{\prime} \boldsymbol{\Lambda}\right) S^{-1}\left(S(x)^{\prime \prime}\right)=\boldsymbol{\lambda}\left(\epsilon\left(S(x)^{\prime}\right) \boldsymbol{\Lambda}\right) S^{-1}\left(S(x)^{\prime \prime}\right)=S^{-1}\left(\epsilon\left(S(x)^{\prime}\right) S(x)^{\prime \prime}\right)=x$, где в третьем равенстве мы воспользовались формулой $(2.3)$. Аналогично $\widehat{\phi}^{-1}(\widehat{\phi}(\beta))=$ $\widehat{\phi}^{-1}\left(\beta\left(\boldsymbol{\Lambda}^{\prime}\right) \boldsymbol{\Lambda}^{\prime \prime}\right) \beta\left(\boldsymbol{\Lambda}^{\prime}\right) \boldsymbol{\lambda}\left(S\left(\boldsymbol{\Lambda}^{\prime \prime}\right) ?\right)=\beta\left(\boldsymbol{\lambda}\left(S\left(\boldsymbol{\Lambda}^{\prime \prime}\right) ?\right) \boldsymbol{\Lambda}^{\prime}\right)=\beta\left(\boldsymbol{\lambda}\left(S(\boldsymbol{\Lambda})^{\prime} ?\right) S^{-1}\left(S(\boldsymbol{\Lambda})^{\prime \prime}\right)\right)=$ $\beta\left(\boldsymbol{\lambda}\left(S(\boldsymbol{\Lambda}) ?^{\prime}\right) ?^{\prime \prime}\right)=\beta\left(\boldsymbol{\lambda}\left(\boldsymbol{\Lambda} ?^{\prime}\right) ?^{\prime \prime}\right)=\beta\left(\boldsymbol{\lambda}\left(\epsilon\left(?^{\prime}\right) \boldsymbol{\Lambda}\right) ?^{\prime \prime}\right)=\beta\left(\epsilon\left(?^{\prime}\right) ?^{\prime \prime}\right)=\beta$, где вновь использовано $(2.3)$.

\footnotetext{
3) Поэтому $U^{*}$ предполагается унимодулярной; для рассматриваемых ниже квантовых групп это выполнено.

4) Мы используем обозначения Свидлера $\Delta(x)=\sum_{(x)} x^{\prime} x^{\prime \prime}$ (см., например, [29]), в большинстве случаев опуская при этом символ суммирования; определяющее свойство интеграла тогда записывается как $\boldsymbol{\lambda}\left(x^{\prime}\right) x^{\prime \prime}=\boldsymbol{\lambda}(x)$.

5) Здесь и далее мы используем определения антипода и коединицы, записанные в виде (см., например, [29]) $x^{\prime} S\left(x^{\prime \prime}\right)=S\left(x^{\prime}\right) x^{\prime \prime}=\epsilon(x) 1$ и $x^{\prime} \epsilon\left(x^{\prime \prime}\right)=\epsilon\left(x^{\prime}\right) x^{\prime \prime}=x$. Тогда, в частности, $x^{\prime} S^{-1}\left(x^{\prime \prime \prime}\right) x^{\prime \prime}=x$.
} 
Покажем, что $\widehat{\phi}$ сплетает структуры левых $U$-модулей на $U^{*}$ и $U$. Нам надо доказать, что $\beta\left(S(x) \boldsymbol{\Lambda}^{\prime}\right) \boldsymbol{\Lambda}^{\prime \prime}=x \beta\left(\boldsymbol{\Lambda}^{\prime}\right) \boldsymbol{\Lambda}^{\prime \prime}$ или, что эквивалентно, $\beta\left(x \boldsymbol{\Lambda}^{\prime}\right) \boldsymbol{\Lambda}^{\prime \prime}=$ $S^{-1}(x) \beta\left(\boldsymbol{\Lambda}^{\prime}\right) \boldsymbol{\Lambda}^{\prime \prime}$. Но мы имеем $\beta\left(x \boldsymbol{\Lambda}^{\prime}\right) \boldsymbol{\Lambda}^{\prime \prime}=\beta\left(\epsilon\left(x^{\prime}\right) x^{\prime \prime} \boldsymbol{\Lambda}^{\prime}\right) \boldsymbol{\Lambda}^{\prime \prime}=\beta\left(\left(x^{\prime} \boldsymbol{\Lambda}\right)^{\prime}\right) S^{-1}\left(x^{\prime \prime}\right) \times$ $\left(x^{\prime} \boldsymbol{\Lambda}\right)^{\prime \prime}=\beta\left(\epsilon\left(x^{\prime}\right) \boldsymbol{\Lambda}^{\prime}\right) S^{-1}\left(x^{\prime \prime \prime}\right) \epsilon\left(x^{\prime \prime}\right) \boldsymbol{\Lambda}^{\prime \prime}=S^{-1}(x) \beta\left(\boldsymbol{\Lambda}^{\prime}\right) \boldsymbol{\Lambda}^{\prime \prime}$.

2.3. Следы и отображение Радфорда. Для любого неприводимого представления $\mathcal{X}$ квантовой группы $U$ (“квантовый”) след, заданный формулой (П.10), является инвариантным функционалом на $U$, т.е. элементом пространства $\mathrm{Ch}(U)$ $(\text { см. п. П.1 })^{6)}$. Но, как уже было замечено, пространство $q$-характеров Ch не порождается $q$-следами по неприводимым модулям. Отображение Радфорда отправляет каждый функционал $\operatorname{Tr}_{\mathcal{X}}\left(\boldsymbol{g}^{-1} ?\right)$ в центр Z алгебры $U$ :

$$
\widehat{\phi}: \operatorname{Tr}_{\mathcal{X}}\left(\boldsymbol{g}^{-1} ?\right) \mapsto \widehat{\phi}(\mathcal{X}) \in \mathrm{Z}
$$

(здесь достаточно, чтобы $\mathcal{X}$ перечислял неприводимые представления $U$, поскольку следы "видят" только неприводимые подфакторы в неразложимых представлениях). Коль скоро линейная оболочка $q$-следов по неприводимым модулям не совпадает со всем пространством $q$-характеров, образы неприводимых представлений при отображении Радфорда не покрывают весь центр.

Отметим следующее свойство отображения Радфорда. Для любого центрального элемента $a \in \mathbf{Z}$ его действие на неприводимом представлении $\mathcal{X}$ дается умножением на скаляр, который мы обозначим как $a_{\mathcal{X}} \in \mathbb{C}$. Тогда имеет место соотношение в центре

$$
a \widehat{\phi}(\mathcal{X})=a_{\mathcal{X}} \widehat{\phi}(\mathcal{X})
$$

В частности, образ всех неприводимых представлений (следов по ним) при отображении Радфорда есть аннулятор радикала в центре.

2.3.1. Для $\overline{\mathcal{U}}_{\mathfrak{q}} s \ell(2)$ несложно проверить, что правый интеграл и двусторонний коинтеграл даются формулами ${ }^{7)}$

$$
\begin{gathered}
\lambda\left(F^{j} E^{m} K^{n}\right)=\frac{1}{\zeta} \delta_{j, p-1} \delta_{m, p-1} \delta_{n, p+1}, \\
\Lambda=\zeta F^{p-1} E^{p-1} \sum_{j=0}^{2 p-1} K^{j}
\end{gathered}
$$

где мы выбираем нормировочный множитель $\zeta=\sqrt{p / 2}([p-1] !)^{-2}$ [9]. Для квантовой группы $\mathcal{U}_{p, p^{\prime}}$ выражения для $\boldsymbol{\lambda}$ и $\boldsymbol{\Lambda}$ в работе [11] также "используют" соответствующий аналог того факта, что имеется старшая степень, в которой внедиагональные генераторы квантовой группы не равны нулю.

6)Читатель, не склонный следить за подробностями определения $\boldsymbol{g}$ в (П.10), может считать его просто тем элементом, который делает след "квантовым", т.е. инвариантным относительно коприсоединенного действия квантовой группы.

7) Вообще говоря, (ко)интеграл определен лишь с точностью до ненулевого множителя, но факторизуемые ленточные квантовые группы предоставляют “каноническую" нормировку, выводимую из условия $\mathcal{S}^{2}=\mathrm{id}$ на центре; в соответствии с $(1.7)$ нормировка $\mathcal{S}$ наследуется из нормировки $\widehat{\phi}$ и поэтому также из нормировки коинтеграла. 
2.4. Комодуль. Еще одно общее понятие, которое нам понадобится - это понятие комодуля (comodulus). Для правого интеграла $\boldsymbol{\lambda}$ комодуль "измеряет", насколько $\boldsymbol{\lambda}$ отличается от левого интеграла (см. [32]): это такой элемент $\boldsymbol{a} \in U$, что $(\mathrm{id} \otimes \boldsymbol{\lambda}) \Delta(x)=\boldsymbol{\lambda}(x) \boldsymbol{a}$ для любого $x \in U$.

Простое вычисление показывает, что $\overline{\mathcal{U}}_{\mathfrak{q}} s \ell(2)$-комодуль $\boldsymbol{a}=K^{2}$. Для $\mathcal{U}_{p, p^{\prime}}$ комодуль есть $\boldsymbol{a}=K^{2 p-2 p^{\prime}}$.

\section{3. КВАНТОВО-ГРУППОВЫЕ МОДУЛИ: ОТ НЕПРИВОДИМЫХ К ПРОЕКТИВНЫМ}

Ниже рассматриваются неприводимые (простые) и проективные представления квантовой группы. В соответствии с общей философией двойственности КажданаЛюстига неприводимые представления квантовой группы некоторым образом "соответствуют" неприводимым представлениям киральной алгебры в логарифмических конформных моделях. В частности, кольцо Гротендика связано с алгеброй слияний в конформной теории поля. Прямое вычисление слияний представлений киральной алгебры является, как правило, весьма трудной задачей, поэтому структуру кольца Гротендика можно (и имеются свидетельства в пользу того, что это не вполне бессмысленно) рассматривать как "слияния для бедных". Помимо неприводимых представлений важную роль играют также их проективные накрывающие. В качестве конкретного примера мы опишем некоторые аспекты теории представлений квантовой группы $\overline{\mathcal{U}}_{\mathfrak{q}} s \ell(2)$.

3.1. Неприводимые представления и кольцо Гротендика. Существуют $2 p$ неприводимых $\overline{\mathcal{U}}_{\mathfrak{q}} s \ell(2)$-представлений $\mathcal{X}_{r}^{ \pm}$, которые удобно нумеровать знаками \pm и индексом $r, 1 \leqslant r \leqslant p$. Вектор старшего веса $|r\rangle^{ \pm}$представления $\mathcal{X}_{r}^{ \pm}$аннулируется оператором $E$, а вес определяется соотношением $K|r\rangle^{ \pm}= \pm \mathfrak{q}^{r-1}|r\rangle^{ \pm}$. Размерность представления есть $\operatorname{dim} \mathcal{X}_{r}^{ \pm}=r$.

Напомним, что кольцо Гротендика - это свободная абелева группа, порожденная символами $[M]$, где $M$ пробегает все представления по модулю соотношений $[M]=$ $\left[M^{\prime}\right]+\left[M^{\prime \prime}\right]$ для каждой точной последовательности $0 \rightarrow M^{\prime} \rightarrow M \rightarrow M^{\prime \prime} \rightarrow 0$. Умножение в кольце индуцируется тензорным произведением представлений, когда всякий неразложимый модуль, возникающий в тензорном произведении, заменяется суммой своих простых подфакторов.

Квантовая группа $\overline{\mathcal{U}}_{\mathfrak{q}} s \ell(2)$. Кольцо Гротендика алгебры $\overline{\mathcal{U}}_{\mathfrak{q}} s \ell(2)$ вычисляется (вполне непосредственно, см. [9]) как

$$
\mathcal{X}_{r}^{\alpha} \mathcal{X}_{s}^{\alpha^{\prime}}=\sum_{\substack{t=|r-s|+1, \operatorname{mar}=2}}^{r+s-1} \widetilde{\mathcal{X}}_{t}^{\alpha \alpha^{\prime}}
$$

где

$$
\widetilde{\mathcal{X}}_{r}^{\alpha}= \begin{cases}\mathcal{X}_{r}^{\alpha}, & 1 \leqslant r \leqslant p \\ \mathcal{X}_{2 p-r}^{\alpha}+2 \mathcal{X}_{r-p}^{-\alpha}, & p+1 \leqslant r \leqslant 2 p-1\end{cases}
$$


Его можно описать также [9] в терминах многочленов Чебышева как фактор кольца многочленов $\mathbb{C}[x]$ по идеалу, порожденному многочленом

$$
\widehat{\Psi}_{2 p}(x)=U_{2 p+1}(x)-U_{2 p-1}(x)-2,
$$

где $U_{s}(x)$ - многочлены Чебышева второго рода:

$$
U_{s}(2 \cos t)=\frac{\sin s t}{\sin t}, \quad s \geqslant 1 .
$$

Они удовлетворяют рекуррентным соотношениям $x U_{s}(x)=U_{s-1}(x)+U_{s+1}(x), s \geqslant 2$, с начальными условиями $U_{1}(x)=1, U_{2}(x)=x$. Более того, пусть

$$
P_{s}(x)= \begin{cases}U_{s}(x), & 1 \leqslant s \leqslant p \\ \left(U_{s}(x)-U_{2 p-s}(x)\right) / 2, & p+1 \leqslant s \leqslant 2 p .\end{cases}
$$

При отображении на фактор образ каждого $P_{s}$ совпадает с $\mathcal{X}_{s}^{+}$при $1 \leqslant s \leqslant p$ и с $\mathcal{X}_{s-p}^{-}$при $p+1 \leqslant s \leqslant 2 p$.

Алгебра (3.1) является неполупростой алгеброй Верлинде (коммутативной ассоциативной алгеброй с неотрицательными целыми структурными коэффициентами в специальном базисе, см. [33]), единица в которой есть $\mathcal{X}_{1}^{+}$. Алгебра содержит идеал $\mathrm{V}_{p+1}$, порожденнный $\mathcal{X}_{p}^{+}, \mathcal{X}_{p}^{-}$и $\mathcal{X}_{p-r}^{+}+\mathcal{X}_{r}^{-}$при $1 \leqslant r \leqslant p-1$. Фактор по $\mathrm{V}_{p+1}$ является полупростой алгеброй Верлинде и в действительности совпадает с алгеброй слияний унитарных $\widehat{s \ell}(2)$-представлений уровня $p-2$.

Эта же алгебра была получена в работе [19] из модулярных преобразований характеров триплетной алгебры в логарифмических $(p, 1)$-моделях в рамках неполупростого обобщения формулы Верлинде (см. также [34] по поводу сравнения с другими способами получить алгебру слияний).

Квантовая группа $\mathcal{U}_{p, p^{\prime}}$ Квантовая группа $\mathcal{U}_{p, p^{\prime}}$, двойственная логарифмической

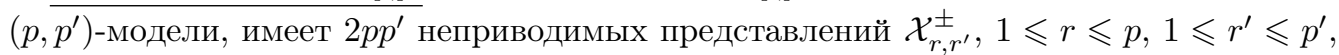
для которых $\operatorname{dim} \mathcal{X}_{r, r^{\prime}}^{ \pm}=r r^{\prime}$. Структура кольца Гротендика имеет вид [11]

$$
\mathcal{X}_{r, r^{\prime}}^{\alpha} \mathcal{X}_{s, s^{\prime}}^{\beta}=\sum_{\substack{u=|r-s|+1, \\ \text { шаг=2 }}}^{r+s-1} \sum_{\substack{u^{\prime}=\left|r^{\prime}-s^{\prime}\right|+1, \\ \text { шаг=2 }}}^{r^{\prime}+s^{\prime}-1} \widetilde{\mathcal{X}}_{u, u^{\prime}}^{\alpha \beta},
$$

где

$$
\widetilde{\mathcal{X}}_{r, r^{\prime}}^{\alpha}= \begin{cases}\mathcal{X}_{r, r^{\prime}}^{\alpha}, & 1 \leqslant r \leqslant p, \quad 1 \leqslant r^{\prime} \leqslant p^{\prime}, \\ \mathcal{X}_{2 p-r, r^{\prime}}^{\alpha}+2 \mathcal{X}_{r-p, r^{\prime}}^{-\alpha}, & p+1 \leqslant r \leqslant 2 p-1, \quad 1 \leqslant r^{\prime} \leqslant p^{\prime}, \\ \mathcal{X}_{r, 2 p^{\prime}-r^{\prime}}^{\alpha}+2 \mathcal{X}_{r, r^{\prime}-p^{\prime}}^{-\alpha}, & 1 \leqslant r \leqslant p, \quad p^{\prime}+1 \leqslant r^{\prime} \leqslant 2 p^{\prime}-1, \\ \mathcal{X}_{2 p-r, 2 p^{\prime}-r^{\prime}}^{\alpha}+2 \mathcal{X}_{2 p-r, r^{\prime}-p^{\prime}}^{\alpha}+ & \\ +2 \mathcal{X}_{r-p, 2 p^{\prime}-r^{\prime}}^{-\alpha}+4 \mathcal{X}_{r-p, r^{\prime}-p^{\prime}}^{\alpha}, & p+1 \leqslant r \leqslant 2 p-1, \quad p^{\prime}+1 \leqslant r^{\prime} \leqslant 2 p^{\prime}-1 .\end{cases}
$$

Эта алгебра является фактором $\mathbb{C}[x, y]$, как это описано в работе [11]. Радикал в этой неполупростой алгебре Верлинде (с единицей, равной $\left.\mathcal{X}_{1,1}^{+}\right)$порожден действием алгебры на $\mathcal{X}_{p, p^{\prime}}^{+}$; фактор по радикалу совпадает с алгеброй слияния минимальной $\left(p, p^{\prime}\right)$-модели для алгебры Вирасоро.

Эта алгебра является вполне реальным кандидатом на алгебру слияний (" $K_{0}$-типа") представлений $W$-алгебры в логарифмических $\left(p, p^{\prime}\right)$-моделях, см. [13], [26]. 


\section{2. Неразложимые модули.}

3.2.1. Неприводимые модули над квантовой группой могут “склеиваться" в неразложимые представления. Уже для $\overline{\mathcal{U}}_{\mathfrak{q}} s \ell(2)$ неразложимые представления (которые были классифицированы вполне непосредственным образом в работе [10] и которые легко также извлечь из более общих результатов работы [35]) достаточно многочисленны. Помимо проективных модулей, которые мы рассмотрим отдельно в п. 3.3, неразложимые представления даются семействами модулей $\mathcal{W}_{r}^{ \pm}(n), \mathcal{M}_{r}^{ \pm}(n)$ и $\mathcal{O}_{r}^{ \pm}(n, z)$, которые можно представить соответственно как

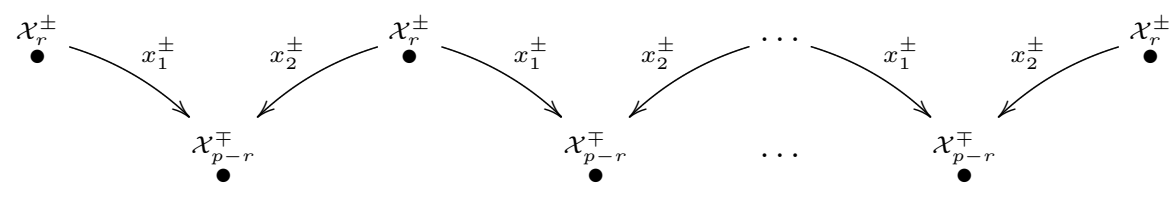

(где $1 \leqslant r \leqslant p-1$, целое $n-$ это число модулей на верхнем этаже, $n \geqslant 2$ ),

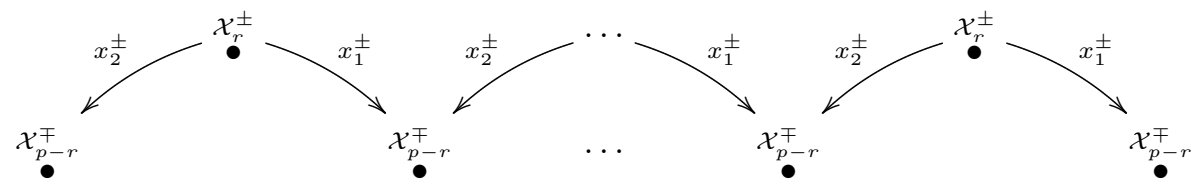

(где $1 \leqslant r \leqslant p-1$, целое $n-$ это число модулей на нижнем этаже, $n \geqslant 2$ ) и

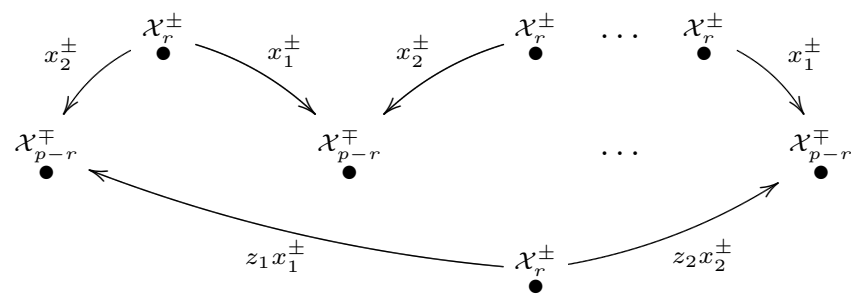

(где $1 \leqslant r \leqslant p-1, z=z_{1}: z_{2} \in \mathbb{C P}^{1}$, целое $n$ - это число $\mathcal{X}_{r}^{ \pm}$-модулей, $n \geqslant 1$ ). Малые $x_{i}^{+}$и $x_{i}^{-}, i=1,2$, являются базисными элементами в соответствующих пространствах $\mathbb{C}^{2}=\operatorname{Ext} \frac{1}{\bar{u}_{\mathfrak{q}} s \ell(2)}\left(\mathcal{X}_{r}^{+}, \mathcal{X}_{p-r}^{-}\right)$и $\mathbb{C}^{2}=\operatorname{Ext} \frac{1}{\bar{u}_{\mathfrak{q}} s \ell(2)}\left(\mathcal{X}_{p-r}^{-}, \mathcal{X}_{r}^{+}\right) ;$они в действительности порождают всю алгебру $\operatorname{Ext}_{s}^{\bullet}$ (с умножением Йонеды) с соотношениями (см. подробности в [10])

$$
x_{i}^{+} x_{j}^{+}=x_{i}^{-} x_{j}^{-}=x_{1}^{+} x_{2}^{-}+x_{2}^{+} x_{1}^{-}=x_{1}^{-} x_{2}^{+}+x_{2}^{-} x_{1}^{+}=0 .
$$

Интересно, что весьма схожая картина (“зигзаг-модули”, хотя и без О-модулей) возникала также в другом контексте в работах [36], [37].

3.2.2. Категория конечномерных представлений разлагается на подкатегории следующим образом. Для $\overline{\mathcal{U}}_{\mathfrak{q}} s \ell(2)$ хорошо известный (“квантовый”) элемент Казимира

$$
\boldsymbol{C}=\left(\mathfrak{q}-\mathfrak{q}^{-1}\right)^{2} E F+\mathfrak{q}^{-1} K+\mathfrak{q} K^{-1}
$$


удовлетворяет минимальному полиномиальному соотношению $\Psi_{2 p}(\boldsymbol{C})=0$, где [9]

$$
\Psi_{2 p}(x)=\left(x-\beta_{0}\right)\left(x-\beta_{p}\right) \prod_{s=1}^{p-1}\left(x-\beta_{s}\right)^{2}, \quad \beta_{s}=\mathfrak{q}^{s}+\mathfrak{q}^{-s} .
$$

Это соотношение определяет разложение категории представлений в прямую сумму полных подкатегорий $\mathfrak{C}(s)$ таких, что $\left(\boldsymbol{C}-\boldsymbol{\beta}_{s}\right)$ действует нильпотентно на объектах из $\mathfrak{C}(s)$. Поскольку $\beta_{s} \neq \beta_{s^{\prime}}$ при $0 \leqslant s \neq s^{\prime} \leqslant p$, имеются $p+1$ полных подкатегорий $\mathfrak{C}(s), 0 \leqslant s \leqslant p$. Каждая $\mathfrak{C}(s)$ при $1 \leqslant s \leqslant p-1$ содержит ровно два неприводимых модуля $\mathcal{X}_{s}^{+}$и $\mathcal{X}_{p-s}^{-}$(поскольку элемент Казимира действует как умножение на $\beta_{s}$ именно на них) и бесконечно много неразложимых модулей, получаемых путем указанных выше склеек. Неприводимые модули $\mathcal{X}_{p}^{+}$и $\mathcal{X}_{p}^{-}$, отвечающие собственным значениям $\beta_{p}$ и $\beta_{0}$, составляют соответствующие категории $\mathfrak{C}(p)$ и $\mathfrak{C}(0)$.

3.3. Проективные модули. Процесс построения расширений останавливается на проективных модулях - проективных накрывающих всех неприводимых представлений. Взятие прямых сумм проективных модулей дает проективные накрывающие любых неразложимых представлений.

Некоторые неприводимые представления являются своими собственными проективными накрывающими: это модули $\mathcal{X}_{p}^{ \pm}$для $\overline{\mathcal{U}}_{\mathfrak{q}} s \ell(2)$ и $\mathcal{X}_{p, p^{\prime}}^{ \pm}$для $\mathcal{U}_{p, p^{\prime}}$. Остальные неприводимые представления имеют проективные накрывающие, фильтруемые несколькими неприводимыми подфакторами.

Для $\overline{\mathcal{U}}_{\mathfrak{q}} s \ell(2)$ проективную накрывающую $\mathcal{P}_{r}^{ \pm}$модуля $\mathcal{X}_{r}^{ \pm}, r=1, \ldots, p-1$, можно представить как ${ }^{8)}$

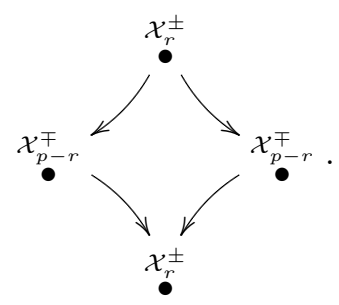

Имеем $\operatorname{dim} \mathcal{P}_{r}^{ \pm}=2 p$. Для $\mathcal{U}_{p, p^{\prime}}$, кроме двух неприводимых проективных модулей размерности $p p^{\prime}$, имеются $2\left(p-1+p^{\prime}-1\right)$ проективных модулей размерности $2 p p^{\prime}$ и $2(p-1)\left(p^{\prime}-1\right)$ проективных модулей размерности $4 p p^{\prime}$ (см. [11], где также приведена диаграмма с шестнадцатью подфакторами).

3.3.1. От кольца Гротендика к тензорной алгебре. Результаты, приведенные в работе [35], выходят за рамки кольца Гротендика для квантовой группы, тесно связанной с $\overline{\mathcal{U}}_{\mathfrak{q}} s \ell(2):$ в этой работе вычислено тензорное произведение неразложимых

${ }^{8)}$ В диаграммах такого типа, во-первых, стрелки направлены к подмодулям; во-вторых, подразумевается, что действие квантовой группы на каждом неприводимом представлении изменено в согласии со стрелками, связывающими данный подфактор с другими. Это, конечно, верно и для рассмотренных выше “двухэтажных" неразложимых модулей, но еще более важно для проективных модулей, в которых $\left(\stackrel{\mathcal{X}_{r}^{ \pm}}{\bullet}{\stackrel{\mathcal{X}_{p}^{\mp}}{\mp}}^{\bullet}\right)$-расширения сами по себе не достаточны для описания действия квантовой группы. Построение действия на проективном модуле требует некоторых усилий, хотя и не является слишком сложным для каждой из рассматриваемых здесь квантовых групп, как это видно из явных формул в работах [9], [11]. 
представлений. Из рассуждений работы [35] следует, что кольцо Гротендика для $\overline{\mathcal{U}}_{\mathfrak{q}} s \ell(2)$ (3.1) является в действительности результатом "насильственного полуупрощения" следующей алгебры тензорных произведений неприводимых представлений. Во-первых, если $r+s-p \leqslant 1$, то, очевидно, в разложении возникают только неприводимые представления:

$$
\mathcal{X}_{r}^{\alpha} \otimes \mathcal{X}_{s}^{\beta}=\bigoplus_{\substack{t=|r-s|+1 \\ \text { шаг=2 }}}^{r+s-1} \mathcal{X}_{t}^{\alpha \beta}
$$

(сумма содержит $\min (r, s)$ слагаемых). Далее, если $r+s-p \geqslant 2$ четно, $r+s-p=2 n$ при $n \geqslant 1$, то

$$
\mathcal{X}_{r}^{\alpha} \otimes \mathcal{X}_{s}^{\beta}=\bigoplus_{\substack{t=|r-s|+1, \\ \text { шаг=2 }}}^{2 p-r-s-1} \mathcal{X}_{t}^{\alpha \beta} \oplus \bigoplus_{a=1}^{n} \mathcal{P}_{p+1-2 a}^{\alpha \beta} .
$$

Наконец, если $r+s-p \geqslant 3$ нечетно, $r+s-p=2 n+1$ при $n \geqslant 1$, то

$$
\mathcal{X}_{r}^{\alpha} \otimes \mathcal{X}_{s}^{\beta}=\bigoplus_{\substack{t=|r-s|+1, \\ \text { шаг }=2}}^{2 p-r-s-1} \mathcal{X}_{t}^{\alpha \beta} \oplus \bigoplus_{a=0}^{n} \mathcal{P}_{p-2 a}^{\alpha \beta}
$$

Заметим, что в каждой из двух последних формул первая сумма в правой части равенства содержит $p-\max (r, s)$ слагаемых, а потому исчезает, если $\max (r, s)=p$ (см. [35] по поводу тензорных произведений других модулей из п. 3.2.1).

3.3.2. Замечания. Обратим внимание на следующие факты.

1. Как мы видим, неприводимые $\overline{\mathcal{U}}_{\mathfrak{q}} s \ell(2)$-представления производят в тензорной алгебре (себя и) проективные модули. Поскольку тензорные произведения любого модуля с проективными разлагаются в проективные модули, можно согласованным образом ограничиться только неприводимыми и проективными модулями (другими словами, имеется подкольцо в тензорной алгебре). Однако это весьма специальная ситуация, специфичная для $\overline{\mathcal{U}}_{\mathfrak{q}} s \ell(2)$ (и слегка большей алгебры, рассмотренной в работе [35]); вообще говоря, отличные от проективных неразложимые представления появляются в тензорных произведениях неприводимых представлений ${ }^{9)}$. В частности, "настоящая" тензорная алгебра, которой отвечает кольцо Гротендика (3.3), по всей видимости, включает различные неразложимые модули в произведении неприводимых представлений. Но уже для $\overline{\mathcal{U}}_{\mathfrak{q}} s \ell(2)$ полное описание тензорной алгебры требует вычисления произведений всех представлений, перечисленых в п. 3.2.1.

2. Повторяя то, что обсуждалось в [10], отметим, что предыдущее замечание в полной мере применимо к алгебре слияния представлений киральной алгебры (триплетной $W$-алгебры [8], [27], [38]) в логарифмических $(p, 1)$-моделях конформной теории, коль скоро слияния берутся не в $K_{0}$-варианте, а с "настоящими" неразложимыми представлениями [24], [25]. Хотя и можно рассматривать такую алгебру слияний только для неприводимых и проективных модулей, полная алгебра слияния должна включать в себя все неразложимые $\mathcal{W}$-, $\mathcal{M}-$-, и $\mathcal{O}$-модули над триплетной алгеброй (причем последние, заметим, несколько интригующим образом зависят от $\left.z \in \mathbb{C P}^{1}\right)$.

\footnotetext{
9) Автор признателен Ф. Шомерусу за это замечание и за обсуждение этого вопроса.
} 
3.3.3. Псевдоследы. Проективные модули служат еще одной несколько технической, но полезной цели. Как уже было отмечено в п. 2.3, следы по неприводимым представлениям не порождают линейно все пространство $q$-характеров Ch. Проективные модули дают то, чего не хватает: они позволяют построить псевдоследы $\operatorname{Tr}_{\mathbb{P}}\left(\boldsymbol{g}^{-1} ? \sigma\right)$ (для некоторых отображений и модулей $\sigma: \mathbb{P} \rightarrow \mathbb{P}$ ), которые вместе со следами $\operatorname{Tr}_{\mathcal{X}}\left(\boldsymbol{g}^{-1} ?\right)$ по неприводимым представлениям линейно порождают всё $\mathrm{Ch}$, т.е. в Ch можно построить такой базис $\gamma_{A}$, что часть $q$-характеров среди $\gamma_{A}$ дается следами по неприводимым представлениям, а остальные - псевдоследами, связанными с проективными модулями в каждой полной подкатегории.

Схема построения псевдоследов такова [11]. Для всякого (приводимого) модуля $\mathbb{P}$ и отображения $\sigma: \mathbb{P} \rightarrow \mathbb{P}$ функционал

$$
\gamma: x \mapsto \operatorname{Tr}_{\mathbb{P}}\left(\boldsymbol{g}^{-1} x \sigma\right)
$$

является $q$-характером, если и только если (ср. с определением (П.2))

$$
0=\gamma(x y)-\gamma\left(S^{2}(y) x\right) \equiv \operatorname{Tr}_{\mathbb{P}}\left(\boldsymbol{g}^{-1} x[y, \sigma]\right) .
$$

Удается найти приводимые неразложимые модули $\mathbb{P}$ и отображения $\sigma$, удовлетворяющие условию (3.8). Для этого в качестве $\mathbb{P}$ надо взять проективный модуль в выбранной полной подкатегории (одной из тех, что содержат более одного модуля). Для $\overline{\mathcal{U}}_{\mathfrak{q}} s \ell(2)$ таковым является прямая сумма

$$
\mathbb{P}_{r}=\mathcal{P}_{r}^{+} \oplus \mathcal{P}_{p-r}^{-}, \quad 1 \leqslant r \leqslant p-1,
$$

а для $\mathcal{U}_{p, p^{\prime}}$ - прямая сумма

$$
\mathbb{P}_{r, r^{\prime}}=\mathcal{P}_{r, r^{\prime}}^{+} \oplus \mathcal{P}_{p-r, r^{\prime}}^{-} \oplus \mathcal{P}_{r, p^{\prime}-r^{\prime}}^{-} \oplus \mathcal{P}_{p-r, p^{\prime}-r^{\prime}}^{+},
$$

а также "граничные" случаи, когда $r=p$ или $r^{\prime}=p^{\prime}$ и сумма содержит два слагаемых (здесь $\mathcal{P}_{r, r^{\prime}}^{ \pm}-$проективная накрывающая неприводимого представления $\mathcal{X}_{r, r^{\prime}}^{ \pm}$). Во всех случаях $\sigma$ представляет собой линейное отображение, которое отправляет нижний модуль в фильтрации каждого проективного модуля в “тот же" модуль на более высоком уровне фильтрации. Такие отображения определены не однозначно (например, они зависят от выбора базисов и, очевидно, от "примеси" более низко лежащих модулей из фильтрации), во всяком случае, вместе со следами по неприводимым представлениям, они позволяют построить базис в Сh. Для $\overline{\mathcal{U}}_{\mathfrak{q}} s \ell(2)$ имеется единственный псевдослед при каждом $r$ из (3.9), получаемый, когда $\sigma$ отправляет нижнюю вершину каждого из ромбов типа (3.6) в верхнюю. Это дает ровно $p-1$ линейно независимых элементов в Ch. Структура для $\mathcal{U}_{p, p^{\prime}}$ несколько богаче, и подсчет происходит следующим образом [11]. В каждом из четырех проективных модулей в (3.10) имеется не одна, а три других копии нижнего подфактора. Двенадцать возникающих таким образом параметров подчинены семи связям, которые вытекают из (3.8). Среди остающихся пяти различных отображений, удовлетворяющих (3.8), имеется ровно одно (отображение в самую верхушку) для каждого из $(p-1)\left(p^{\prime}-1\right) / 2$ модулей вида $(3.10)$, два отображения для каждого из $(p-1) p^{\prime} / 2$ модулей и еще два отображения для каждого из $p\left(p^{\prime}-1\right) / 2$ модулей. Всего это дает 
$(p-1)\left(p^{\prime}-1\right) / 2+(p-1) p^{\prime}+p\left(p^{\prime}-1\right)$ линейно независимых псевдоследов. Таким образом, вместе со следами по $2 p p^{\prime}$ неприводимым представлениям мы получаем $(3 p-1)\left(3 p^{\prime}-1\right) / 2$ линейно независимых элементов в Ch.

3.3.4. "Базис Радфорда". Образы базиса $\gamma_{A}$ следов и псевдоследов в Ch при отображении Радфорда дают базис $\widehat{\phi}_{A}=\widehat{\phi}\left(\gamma_{A}\right)$ в центре квантовой группы Z. Этот базис играет важную роль ниже, он является одним из двух специальных базисов, связанных преобразованием $\mathcal{S} \in S L(2, \mathbb{Z})$. Другой специальный базис связан с отображением Дринфельда, которое рассматривается в следующем разделе.

3.3.5. Проективные модули и центр. Проективные модули также составляют ключевой ингредиент для нахождения центра квантовой группы (безотносительно к конструкции псевдоследов). Центральные элементы находятся в взаимно однозначном соответствии с эндоморфизмами регулярного представления как бимодуля. Напомним, что рассматриваемое как левый модуль, регулярное представление разлагается на проективные модули, причем каждый из них входит в разложение с кратностью, равной размерности его простого фактора. Обобщение этой картины на разложение бимодуля показывает, что кратности являются в действительности тензорными множителями по отношению к правому действию. Типичный блок бимодульного разложения регулярного представления выглядит следующим образом: по отношению к левому действию он является суммой проективных модулей в одной полной подкатегории, причем каждый из проективных модулей (внешне) тензорно умножен на некий простой модуль. В $\overline{\mathcal{U}}_{\mathfrak{q}} s \ell(2)$ подфакторов немного, и поэтому картина не слишком запутанная, ее можно изобразить в виде [9]
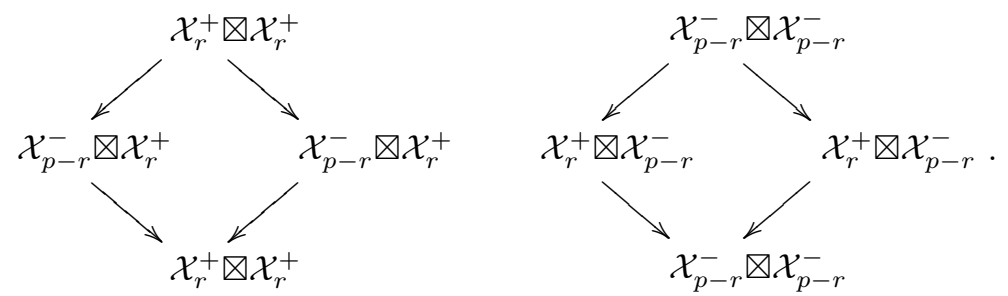

По отношению к правому действию картина полностью симметрична, но при выбранной расстановке подфакторов структуру их расширения приходится изображать как

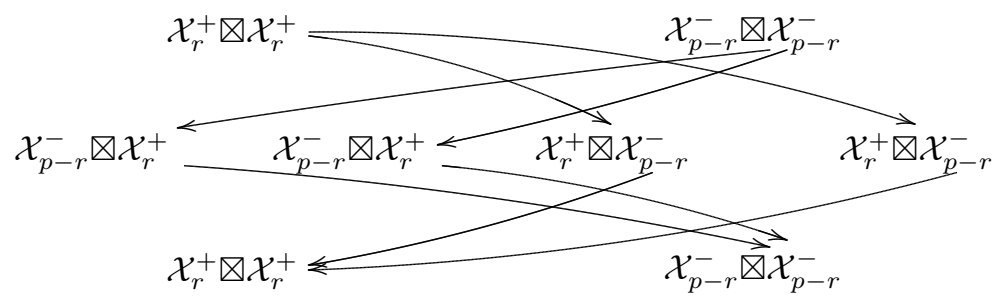

Картинки такого типа немедленно дают число центральных элементов и структуру порождаемой ими ассоциативной алгебры. Во-первых, каждый блок дает один примитивный идемпотент $\boldsymbol{e}_{I}$, являющийся просто проектором на этот блок; 
во-вторых, имеются отображения, отправляющие $A \otimes B$ в "них же" на более низких уровнях, что дает нильпотентные центральные элементы. Для $\overline{\mathcal{U}}_{\mathfrak{q}} s \ell(2)$ бимодульное разложение содержит $p-1$ блок указанной структуры и еще два блока простого вида $\mathcal{X}_{p}^{+} \otimes \mathcal{X}_{p}^{+}$и $\mathcal{X}_{p}^{-} \otimes \mathcal{X}_{p}^{-}$; в каждом из “сложных" блоков имеются два бимодульных автоморфизма, под действием которых верхний блок $\mathcal{X}_{r}^{+} \otimes \mathcal{X}_{r}^{+}$или верхний блок $\mathcal{X}_{p-r}^{-} \otimes \mathcal{X}_{p-r}^{-}$отправляется в соответствующий нижний модуль, что дает два центральных элемента $\boldsymbol{w}_{r}^{+}$и $\boldsymbol{w}_{r}^{-}$со всеми нулевыми произведениями между ними. Поэтому $(3 p-1)$-мерный центр разлагается в прямую сумму ассоциативных алгебр как

$$
\mathrm{Z}_{\overline{\mathfrak{U}}_{\mathfrak{q}} s \ell(2)}=\mathfrak{I}_{p}^{(1)} \oplus \mathfrak{I}_{0}^{(1)} \oplus \bigoplus_{r=1}^{p-1} \mathfrak{B}_{r}^{(3)},
$$

где размерность каждой алгебры указана в качестве верхнего индекса.

Для $\mathcal{U}_{p, p^{\prime}}$ имеется несколько промежуточных уровней в фильтрации проективных модулей, поэтому существует больше нильпотентных элементов, и они имеют нетривиальную таблицу умножения (см. подробности в [11]); центр оказывается $\frac{1}{2}(3 p-1)\left(3 p^{\prime}-1\right)$-мерным и разлагается в прямую сумму ассоциативных алгебр:

$$
\mathrm{Z}_{\mathfrak{u}_{p, p^{\prime}}}=\mathfrak{I}_{p, p^{\prime}}^{(1)} \oplus \mathfrak{I}_{0, p^{\prime}}^{(1)} \oplus \bigoplus_{r=1}^{p-1} \mathfrak{B}_{r, p^{\prime}}^{(3)} \oplus \bigoplus_{r^{\prime}=1}^{p^{\prime}-1} \mathfrak{B}_{p, r^{\prime}}^{(3)} \oplus \bigoplus_{r, r^{\prime} \in \mathcal{I}} \mathfrak{A}_{r, r^{\prime}}^{(9)}
$$

где размерность каждой алгебры указана в качестве верхнего индекса (и где $\left|\mathcal{I}_{1}\right|=$ $\left.(p-1)\left(p^{\prime}-1\right) / 2\right)$. Алгебры $\mathfrak{B}^{(3)}$ в точности такие же, как в предыдущей формуле, а каждая алгебра $\mathfrak{A}_{r, r^{\prime}}^{(9)}$ линейно порождается примитивным идемпотентом $\boldsymbol{e}_{r, r^{\prime}}$ (действующим как единица на всей $\left.\mathfrak{A}_{r, r^{\prime}}^{(9)}\right)$ и восемью элементами из радикала $\boldsymbol{v}_{r, r^{\prime}}^{\nearrow}, \boldsymbol{v}_{r, r^{\prime}}^{\swarrow}$, $\boldsymbol{v}_{r, r^{\prime}}^{\searrow}, \boldsymbol{v}_{r, r^{\prime}}^{\searrow}, \boldsymbol{w}_{r, r^{\prime}}^{\uparrow}, \boldsymbol{w}_{r, r^{\prime}}^{\rightarrow}, \boldsymbol{w}_{r, r^{\prime}}^{\downarrow}, \boldsymbol{w}_{r, r^{\prime}}^{\overleftarrow{ }}$, ненулевые произведения которых имеют вид

$$
\begin{aligned}
& \boldsymbol{v}_{r, r^{\prime}}^{\nearrow} \boldsymbol{v}_{r, r^{\prime}}^{\nwarrow}=\boldsymbol{w}_{r, r^{\prime}}^{\uparrow}, \quad \boldsymbol{v}_{r, r^{\prime}}^{\nearrow} \boldsymbol{v}_{r, r^{\prime}}^{\searrow}=\boldsymbol{w}_{r, r^{\prime}}^{\longrightarrow}, \\
& \boldsymbol{v}_{r, r^{\prime}}^{\swarrow} \boldsymbol{v}_{r, r^{\prime}}^{\nwarrow}=\boldsymbol{w}_{r, r^{\prime}}^{\leftarrow}, \quad \boldsymbol{v}_{r, r^{\prime}}^{\swarrow} \boldsymbol{v}_{r, r^{\prime}}^{\searrow}=\boldsymbol{w}_{r, r^{\prime}}^{\downarrow}
\end{aligned}
$$

\section{4. ОТОБРАЖЕНИЕ ДРИНФЕЛЬДА И ФАКТОРИЗУЕМАЯ И ЛЕНТОЧНАЯ СТРУКТУРЫ}

4.1. $M$-матрица и отображение Дринфельда. Для квазитреугольной алгебры Хопфа $U$ с универсальной $R$-матрицей $M$-матрица является "квадратом" $R$-матрицы и определяется как

$$
M=R_{21} R_{12} \in U \otimes U
$$

Она удовлетворяет соотношениям

$$
(\Delta \otimes \mathrm{id})(M)=R_{32} M_{13} R_{23}, \quad M \Delta(x)=\Delta(x) M \quad \forall x \in U .
$$

Действительно, используя (П.5), находим, что $(\Delta \otimes \mathrm{id})\left(R_{21}\right)=R_{32} R_{31}$, а тогда, используя (П.4), получаем первое соотношение в (4.1). Далее, из (П.3) следует, что $R_{21} R_{12} \Delta(x)=\left(R_{12} \Delta(x)\right)^{\mathrm{op}} R_{12}=\left(\Delta^{\mathrm{op}}(x) R_{12}\right)^{\mathrm{op}} R_{12}=\Delta(x) R_{21} R_{12}$, т.е. второе соотношение в (4.1). 
Отображение Дринфельда $\chi: U^{*} \rightarrow U$ определяется как

$$
\chi: \beta \mapsto(\beta \otimes \mathrm{id})(M),
$$

другими словами, если записать $M$-матрицу в виде

$$
M=\sum_{I} \boldsymbol{m}_{I} \otimes \boldsymbol{n}_{I}
$$

то $\chi(\beta)=\sum_{I} \beta\left(\boldsymbol{m}_{I}\right) \boldsymbol{n}_{I}$.

Если $\chi: U^{*} \rightarrow U$ есть изоморфизм векторных пространств, то алгебра Хопфа $U$ называется факторизуемой [39]. Это означает, что $\boldsymbol{m}_{I}$ и $\boldsymbol{n}_{I}$ в (4.2) являются двумя базисами в $U$.

4.1.1. Лемма [40]. В факторизуемой алгебре Хопфа $U$ ограничение отображения Дринфельдан на $\mathrm{Ch}$ (см $n$. П.1) определяет гомоморфизм $\mathrm{Ch}(U) \rightarrow \mathrm{Z}(U)$ ассочиативных алгебр.

ДоказАтельство. Сначала покажем, что отображение $\chi(\beta)$ центрально для любого элемента $\beta \in \mathrm{Ch}$. Имеем $\chi(\beta) x=\sum_{I} \beta\left(\boldsymbol{m}_{I}\right) \boldsymbol{n}_{I} x=\sum_{I} \beta\left(\boldsymbol{m}_{I} x^{\prime \prime} S^{-1}\left(x^{\prime}\right)\right) \boldsymbol{n}_{I} x^{\prime \prime \prime}$ при $x \in U$. Поскольку $M \Delta(x)=\Delta(x) M$ и $\beta(x y)=\beta\left(S^{2}(y) x\right)$, получаем, что $\chi(\beta) x=\sum_{I} \beta\left(S\left(x^{\prime}\right) x^{\prime \prime} \boldsymbol{m}_{I}\right) x^{\prime \prime \prime} \boldsymbol{n}_{I}=x \boldsymbol{\chi}(\beta)$.

Покажем далее, что $\chi: \mathrm{Ch} \rightarrow \mathrm{Z}$ является гомоморфизмом ассоциативных алгебр; напомним, что произведение двух функционалов определяется как $\beta \gamma(x)=(\beta \otimes$ $\gamma)(\Delta(x))$. Поэтому, используя первое соотношение в $(4.1)$, имеем $\chi(\beta \gamma)=(\beta \gamma \otimes$ id $) M=(\beta \otimes \gamma \otimes \mathrm{id})((\Delta \otimes \mathrm{id})(M))=(\beta \otimes \gamma \otimes \mathrm{id})\left(R_{32} M_{13} R_{23}\right)=(\gamma \otimes \mathrm{id})\left(R_{21} \chi(\beta) R_{12}\right)=$ $\chi(\beta)(\gamma \otimes \mathrm{id})\left(R_{21} R_{12}\right)=\chi(\beta)(\gamma \otimes \mathrm{id})(M)=\chi(\beta) \chi(\gamma)^{10)}$.

Для рассматриваемых здесь квантовых групп указанный выше гомоморфизм является в действительности изоморфизмом (ср. с [40], [41]).

4.2. Двойственные по Каждану-Люстигу квантовые группы: дубль Дринфельда, $R$-матрица и $M$-матрица. Квантовые группы $U$, происходящие из логарифмических конформных моделей, не являются квазитреугольными, но оказываются тем не менее факторизуемыми в следующем смысле: $M$-матрица выражается через некую $R$, которая является универсальной $R$-матрицей для несколько большей квантовой группы $\bar{D}^{11)}$. Это верно и для $\overline{\mathcal{U}}_{\mathfrak{q}} s \ell(2)$, и для $\mathcal{U}_{p, p^{\prime}}$, причем расширение до $\bar{D}$ осуществляется в каждом случае с помощью введения генератора $k=K^{1 / 2}$. Другими словами, в каждом случае имеется квазитреугольная квантовая группа $\bar{D}$ с набором генераторов $k, \ldots$, обладающая универсальной $R$-матрицей $R$, для которой $R_{21} R_{12}$ оказывается лежащим в $U \otimes U$, где $U$ - подалгебра Хопфа в $\bar{D}$, порожденная элементом $K=k^{2}$ и остальными генераторами $\bar{D}$. В наших двух случаях $U-$ это $\overline{\mathcal{U}}_{\mathfrak{q}} s \ell(2)$ или $\mathcal{U}_{p, p^{\prime}}$.

\footnotetext{
10) В работе [41] было отмечено, что “данное Дринфельдом доказательство утверждения [40, 3.3] показывает больше, чем утверждается в [40, 3.3]". Действительно, видно, что $\chi(\beta \gamma)=\chi(\beta) \chi(\gamma)$ если $\beta \in \mathrm{Ch}(U)$ и $\gamma \in U^{*}$.

11) Стандартное определение факторизуемой квантовой группы [39] включает и универсальную $R$-матрицу, поэтому мы проявляем известную осторожность; $R$-матрица в $M$-матричном свойстве (4.1) не является элементом $U \otimes U$. В частности, в нашем случае $U$ не унимодулярна (но $U^{*}$ унимодулярна!).
} 
Вид универсальной $R$-матрицы для $\bar{D}$ в свою очередь следует из конструкции дубля Дринфельда [42] квантовой группы $B$, порожденной скринингами в логарифмической конформной модели [9], $[13]^{12)}$. Для $(p, 1)$-моделей $B$ является тафтовой алгеброй Хопфа с генераторами $E$ и $k$, для которых $k E k^{-1}=\mathfrak{q} E, E^{p}=0$ и $k^{4 p}=1$. Далее рассматривается двойственное пространство $B^{*}$, которое представляет собой алгебру Хопфа с умножением, коумножением, единицей, коединицей и антиподом, задаваемыми как

$$
\begin{gathered}
\langle\beta \gamma, x\rangle=\left\langle\beta, x^{\prime}\right\rangle\left\langle\gamma, x^{\prime \prime}\right\rangle, \quad\langle\Delta(\beta), x \otimes y\rangle=\langle\beta, y x\rangle, \\
\langle 1, x\rangle=\epsilon(x), \quad \epsilon(\beta)=\langle\beta, 1\rangle, \quad\langle S(\beta), x\rangle=\left\langle\beta, S^{-1}(x)\right\rangle
\end{gathered}
$$

при $\beta, \gamma \in B^{*}$ и $x, y \in B$. Дубль Дринфельда $D(B)$ является алгеброй Хопфа на векторном пространстве $B^{*} \otimes B$ с умножением, коумножением, единицей, коединицей и антиподом, определяемыми соответствующими структурами в $B$, формулами (4.3), а также формулой

$$
x \beta=\beta\left(S^{-1}\left(x^{\prime \prime \prime}\right) ? x^{\prime}\right) x^{\prime \prime}, \quad x \in B, \quad \beta \in B^{*} .
$$

Получающаяся в результате алгебра Хопфа $D(B)$ канонически снабжается универсальной $R$-матрицей [42].

Процедура взятия дубля вводит также элемент $\varkappa$, двойственный картановскому элементу $k$, который надо далее исключить переходом к фактору по хопфову идеалу, порожденному элементом $k \varkappa-1$ (в дубле $k \varkappa$ оказывается центральным). Фактор $\bar{D}$ по-прежнему является квазитреугольным, но вычисление $M$-матрицы и ленточного элемента для него показывает, что они в действительности оказываются соответственно $M$-матрицей и ленточным элементом для (хопфовой) подалгебры, порожденной элементом $K \equiv k^{2}$ и остальными генераторами $\bar{D}$, что и дает, наконец, двойственную по Каждану-Люстигу квантовую группу. Именно таким способом эти квантовые группы вместе с ключевыми структурами на них и были получены в [9], [13].

Например, для $\overline{\mathcal{U}}_{\mathfrak{q}} s \ell(2) \quad M$-матрица явно выражается в терминах ПБВ-базиса:

$$
\begin{aligned}
M=\frac{1}{2 p} & \sum_{m=0}^{p-1} \sum_{n=0}^{p-1} \sum_{i=0}^{2 p-1} \sum_{j=0}^{2 p-1} \frac{\left(\mathfrak{q}-\mathfrak{q}^{-1}\right)^{m+n}}{[m] ![n] !} \mathfrak{q}^{m(m-1) / 2+n(n-1) / 2} \times \\
& \times \mathfrak{q}^{-m^{2}-m j+2 n j-2 n i-i j+m i} F^{m} E^{n} K^{j} \otimes E^{m} F^{n} K^{i} .
\end{aligned}
$$

\section{3. Образы следов и псевдоследов при отображении Дринфельда.} В факторизуемой алгебре Хопфа образы следов по неприводимым представлениям при отображении Дринфельда образуют алгебру, изоморфную кольцу Гротендика. Таким образом, имеются центральные элементы

$$
\chi_{r}^{ \pm}=\chi\left(\operatorname{Tr}_{\mathcal{X}_{r}^{ \pm}}\left(\boldsymbol{g}^{-1} ?\right)\right), \quad 1 \leqslant r \leqslant p
$$

12) Скрининги порождают только верхнетреугольную подалгебру в двойственной по Каждану-Люстигу квантовой группе; к этой верхнетреугольной подалгебре добавляются генераторы Картана, построенные из нулевых мод свободных полей, вовлеченных в выбранную свободно-полевую реализацию. Это и дает квантовую группу $B$. 
для $\overline{\mathcal{U}}_{\mathfrak{q}} s \ell(2)$ и центральные элементы

$$
\chi_{r, r^{\prime}}^{ \pm}=\chi\left(\operatorname{Tr}_{\mathcal{X}_{r, r^{\prime}}^{ \pm}}\left(\boldsymbol{g}^{-1} ?\right)\right), \quad 1 \leqslant r \leqslant p, \quad 1 \leqslant r^{\prime} \leqslant p^{\prime},
$$

для $\mathcal{U}_{p, p^{\prime}}$, которые удовлетворяют соответствующим алгебрам (3.1) и (3.3).

4.3.1. Для $\overline{\mathcal{U}}_{\mathfrak{q}} s \ell(2)$ равенство (4.5) позволяет явно вычислить $\chi_{s}^{\alpha}$ :

$$
\begin{gathered}
\chi_{s}^{\alpha}=\alpha^{p+1}(-1)^{s+1} \sum_{n=0}^{s-1} \sum_{m=0}^{n}\left(\mathfrak{q}-\mathfrak{q}^{-1}\right)^{2 m} \mathfrak{q}^{-(m+1)(m+s-1-2 n)} \times \\
\times\left[\begin{array}{c}
s-n+m-1 \\
m
\end{array}\right]\left[\begin{array}{c}
n \\
m
\end{array}\right] E^{m} F^{m} K^{s-1+\beta p-2 n+m},
\end{gathered}
$$

где $\beta=0$, если $\alpha=+1$, и $\beta=1$, если $\alpha=-1$. В частности, $\boldsymbol{\chi}_{2}^{+}=-\boldsymbol{C}$, где $\boldsymbol{C}$ - элемент Казимира (3.4). Тот факт, что элементы $\boldsymbol{\chi}_{r}^{\alpha}$, определяемые формулой (4.6), удовлетворяют соотношениям кольца Гротендика (3.1), приводит к некоторому нетривиальному $q$-биномиальному тождеству, см. [9].

4.3.2. Замечание. Элемент Казимира для $\overline{\mathcal{U}}_{\mathfrak{q}} s \ell(2)$ удовлетворяет минимальному полиномиальному соотношению $\Psi_{2 p}(\boldsymbol{C})=0$, где $\Psi_{2 p}$ определено в (3.5). Это соотношение, в котором $p-1$ корней многочлена $\Psi_{2 p}$ имеют кратность 2 , позволяет построить базис в центре Z алгебры $\overline{\mathcal{U}}_{\mathfrak{q}} s \ell(2)$ из примитивных идемпотентов $\boldsymbol{e}_{r}$ и элементов $\boldsymbol{w}_{r}$ радикала ассоциативной коммутативной алгебры Z [9] (см. [23], а также [43], гл. V.2). Для этого определим многочлены

$$
\begin{gathered}
\psi_{0}(x)=\left(x-\beta_{p}\right) \prod_{r=1}^{p-1}\left(x-\beta_{r}\right)^{2}, \quad \psi_{p}(x)=\left(x-\beta_{0}\right) \prod_{r=1}^{p-1}\left(x-\beta_{r}\right)^{2}, \\
\psi_{s}(x)=\left(x-\beta_{0}\right)\left(x-\beta_{p}\right) \prod_{\substack{r=1, r \neq s}}^{p-1}\left(x-\beta_{r}\right)^{2}, \quad 1 \leqslant s \leqslant p-1,
\end{gathered}
$$

где, напомним, все $\beta_{j}$ попарно различны. Тогда канонические элементы в радикале алгебры Z имеют вид $\boldsymbol{w}_{s}^{ \pm}=\pi_{s}^{ \pm} \boldsymbol{w}_{s}, 1 \leqslant s \leqslant p-1$, где $\boldsymbol{w}_{s}=\left(\boldsymbol{C}-\beta_{s}\right) \psi_{s}(\boldsymbol{C}) / \psi_{s}\left(\beta_{s}\right)$, и мы ввели проекторы

$$
\pi_{s}^{+}=\frac{1}{2 p} \sum_{n=0}^{s-1} \sum_{j=0}^{2 p-1} \mathfrak{q}^{(2 n-s+1) j} K^{j}, \quad \pi_{s}^{-}=\frac{1}{2 p} \sum_{n=s}^{p-1} \sum_{j=0}^{2 p-1} \mathfrak{q}^{(2 n-s+1) j} K^{j}
$$

Канонические центральные идемпотенты определяются как

$$
\boldsymbol{e}_{s}=\frac{1}{\psi_{s}\left(\beta_{s}\right)}\left(\psi_{s}(\boldsymbol{C})-\psi_{s}^{\prime}\left(\beta_{s}\right) \boldsymbol{w}_{s}\right), \quad 0 \leqslant s \leqslant p,
$$

где мы формально полагаем $\boldsymbol{w}_{0}=\boldsymbol{w}_{p}=0$. Аналогичная конструкция имеет место для центра квантовой группы $\mathcal{U}_{p, p^{\prime}}[11]$, где, в частности, существуют не два, а четыре вида проекторов $\pi_{r, r^{\prime}}^{\uparrow}, \pi_{r, r^{\prime}}^{\leftarrow}, \pi_{r, r^{\prime}}, \pi_{r, r^{\prime}}^{\downarrow}$. Для каждой из квантовых групп они представляют собой проекторы на веса, встречающиеся в неприводимых модулях в полной подкатегории, соответствующей нижним индексам. 
4.3.3. "Базис Дринфельда". При применении к базису $\gamma_{A}$ следов и псевдоследов в Ch отображение Дринфельда дает базис $\chi_{A}=\chi\left(\gamma_{A}\right)$ в центре Z. Этот "базис Дринфельда" (который определен неоднозначно, коль скоро псевдоследы определены неоднозначно) задает явное разложение ассоциативной коммутативной алгебры Z на кольцо Гротендика и его линейное дополнение. Произведениям элементов кольца Гротендика с элементами из дополнения также можно придать определенный смысл в контексте соответствия Каждана-Люстига. Полная алгебра $q$-характеров (следов и псевдоследов) для $\overline{\mathcal{U}}_{\mathfrak{q}} s \ell(2)$, отправленная в центр отображением Дринфельда, вычислена в [44]; ее можно понимать как обобщенные слияния, которые интересно сравнить с недавними сходными вычислениями в логарифмических $(p, 1)$-моделях в работе [34].

Очевидно, что под действием отображения $\mathcal{S} \in S L(2, \mathbb{Z})$, определенного в (1.7), "базис Дринфельда" отображается в "базис Радфорда":

$$
\mathcal{S}: \chi_{A} \mapsto \widehat{\phi}_{A} \text {. }
$$

Реализация $\mathcal{T} \in S L(2, \mathbb{Z})$ на центре требует еще одной структуры - ленточного элемента.

4.4. Ленточная структура. Ленточный (ribbon) элемент [45] - это такой элемент $\boldsymbol{v} \in \mathrm{Z}$, для которого $\Delta(\boldsymbol{v})=M^{-1}(\boldsymbol{v} \otimes \boldsymbol{v}), \epsilon(\boldsymbol{v})=1, S(\boldsymbol{v})=\boldsymbol{v}$ (и также $\boldsymbol{v}^{2}=\boldsymbol{u} S(\boldsymbol{u})$, см. п. П.3). Процедура нахождения ленточного элемента включает два шага: сначала находим канонический элемент (П.7) (для чего используется универсальная $R$-матрица для большей квазитреугольной квантовой группы $\bar{D}$, упомянутой в п. 4.2), а далее вычисляем balancing элемент $\boldsymbol{g}$ (см. п. П.4) в соответствии с леммой Дринфельда (П.11), пользуясь комодулем, в свою очередь полученным из явного выражения для интеграла (в этом и состоит участие комодуля во всей схеме). Тогда

$$
\boldsymbol{v}=\boldsymbol{u} \boldsymbol{g}^{-1}
$$

Снова оказывается, что $\boldsymbol{v}$ - элемент хопфовой подалгебры в $\bar{D}$, которая есть $\overline{\mathcal{U}}_{\mathfrak{q}} s \ell(2)$ или $\mathcal{U}_{p, p^{\prime}}$.

4.4.1. Для $\overline{\mathcal{U}}_{\mathfrak{q}} s \ell(2)$, где $\boldsymbol{g}=K^{p+1}$, имеем [9]

$$
\boldsymbol{v}=\sum_{s=0}^{p}(-1)^{s+1} \mathfrak{q}^{-\left(s^{2}-1\right) / 2} \boldsymbol{e}_{s}+\sum_{s=1}^{p-1}(-1)^{p} \mathfrak{q}^{-\left(s^{2}-1\right) / 2}[s] \frac{\mathfrak{q}-\mathfrak{q}^{-1}}{\sqrt{2 p}} \widehat{\boldsymbol{\varphi}}_{s},
$$

где $\boldsymbol{e}_{s}$ - канонические идемпотенты в центре, а

$$
\widehat{\boldsymbol{\varphi}}_{s}=\frac{p-s}{p} \widehat{\boldsymbol{\phi}}_{s}^{+}-\frac{s}{p} \widehat{\boldsymbol{\phi}}_{p-s}^{-}, \quad 1 \leqslant s \leqslant p-1
$$

суть нильпотентные центральные элементы, выражающиеся через $\widehat{\phi}_{s}^{ \pm}$- образы неприводимых представлений $\mathcal{X}_{s}^{ \pm}$(следов по ним) при отображении Радфорда.

4.4.2. Замечание. Из приведенного вида $\boldsymbol{v}$ следует [10], что $\boldsymbol{v}=e^{2 i \pi L_{0}}$ (где $L_{0}-$ нулевая мода в алгебре Вирасоро из логарифмической конформной $(p, 1)$-модели); в частности, содержащие $s^{2}$ показатели степени в (4.8) простым образом связаны 
с конформными размерностями примарных полей. Любопытно, что неполупростое действие $L_{0}$ на решеточной вертекс-операторной алгебре, входящей в конструкцию логарифмической $(p, 1)$-модели, тем самым коррелировано с разложением ленточного элемента по центральным идемпотентам и нильпотентам.

4.4.3. Для $\mathcal{U}_{p, p^{\prime}}$ ленточный элемент имеет вид

$$
\boldsymbol{v}=\sum_{\left(r, r^{\prime}\right) \in \mathcal{I}} e^{2 i \pi \Delta_{r, r^{\prime}}} \boldsymbol{e}_{r, r^{\prime}}+\text { нильпотентные слагаемые, }
$$

где элементы $\boldsymbol{e}_{r, r^{\prime}}$, нумеруемые некоторым множеством индексов $\mathcal{I}$, представляют собой $(p+1)\left(p^{\prime}+1\right) / 2$ примитивных идемпотентов в ассоциативной коммутативной алгебре Z (явный вид нильпотентных слагаемых не добавляет выразительности на принятом уровне детализации, полную формулу см. в [11]), а

$$
\Delta_{r, r^{\prime}}=\frac{\left(p r^{\prime}-p^{\prime} r\right)^{2}-\left(p-p^{\prime}\right)^{2}}{4 p p^{\prime}}
$$

- конформные размерности примарных полей из логарифмической модели [13].

\section{5. ДЕЙСТВИЕ МОДУЛЯРНОЙ ГРУППЫ}

5.1. Определение действия. При определении действия модулярной группы на центре мы следуем работам [21]-[23] с незначительными вариациями в определении $\mathcal{T}$. Эти вариации введены в [9], [13] для упрощения сравнения с представлением модулярной группы, порожденным из характеров киральной алгебры в соответствующих логарифмических конформных моделях. На центре квантовой группы действие $S L(2, \mathbb{Z})$ определяется формулами

$$
\mathcal{S}: x \mapsto \widehat{\phi}\left(\chi^{-1}(x)\right), \quad \mathcal{T}: x \mapsto e^{-i \pi c / 12} \mathcal{S}\left(\boldsymbol{v} \mathcal{S}^{-1}(x)\right),
$$

где $c$ - центральный заряд конформной модели; например, для $\left(p, p^{\prime}\right)$-модели ${ }^{13)} c=$ $13-6 p / p^{\prime}-6 p^{\prime} / p$.

5.2. Результаты вычислений. Результат вычисления отображений (5.1) в каждом случае дает структуру $S L(2, \mathbb{Z})$-представления такого типа, какой был впервые замечен в работе [23] для малой квантовой группы $s \ell(2)^{14)}$.

5.2.1. На центре $\overline{\mathcal{U}}_{\mathfrak{q}} s \ell(2) S L(2, \mathbb{Z})$-представление имеет структуру [9]

$$
\mathrm{Z}_{\bar{u}_{\mathfrak{q}} s \ell(2)}=R_{p+1} \oplus \mathbb{C}^{2} \otimes R_{p-1},
$$

где $\mathbb{C}^{2}$ - определяющее двумерное представление, $R_{p-1}$ есть $(p-1)$-мерное $S L(2, \mathbb{Z})$ представление ("sin $\frac{\pi r s}{p}$-представление", в действительности, представление на унитарных $\widehat{s \ell}(2)_{k}$-характерах уровня $\left.k=p-2\right), R_{p+1}$ есть $(p+1)$-мерное "cos $\frac{\pi r s}{p}$-представление".

\footnotetext{
13) Обращая логику рассуждений, в случае факторизуемой ленточной квантовой группы, для которой можно ожидать ее соответствия с некоторой моделью конформной теории поля, нормировка $\mathcal{T}$ (т.е. множитель, сопровождающий ленточный элемент) может указывать на значение центрального заряда, а разложение ленточного элемента по базису примитивных идемпотентов и элементов из радикала подсказывает конформные размерности.

14) Малые квантовые группы были предметом некоторого внимания в последнее время (см., например, [46], [47] и приведенную там библиографию).
} 
На центре $\mathcal{U}_{p, p^{\prime}} S L(2, \mathbb{Z})$-представление имеет вид [11]

$$
\mathrm{Z}_{\mathrm{u}_{p, p^{\prime}}}=R_{\text {min }} \oplus R_{\text {proj }} \oplus \mathbb{C}^{2} \otimes\left(R_{\square} \oplus R_{\square}\right) \oplus \mathbb{C}^{3} \otimes R_{\text {min }},
$$

где $\mathbb{C}^{3}$ - симметризованный квадрат $\mathbb{C}^{2}, R_{\min }$ есть $\frac{1}{2}(p-1)\left(p^{\prime}-1\right)$-мерное $S L(2, \mathbb{Z})$ представление на характерах рациональной вирасоровской $\left(p, p^{\prime}\right)$-модели, а $R_{\text {proj }}$, $R_{\square}$ и $R_{\square}-$ некоторые $S L(2, \mathbb{Z})$-представления соответствующих размерностей $(p+1)\left(p^{\prime}+1\right) / 2,(p+1)\left(p^{\prime}-1\right) / 2$ и $(p-1)\left(p^{\prime}+1\right) / 2$.

Как уже отмечалось, $(5.2)$ и (5.3) совпадают с соответствующими $S L(2, \mathbb{Z})$-представлениями на обобщенных характерах логарифмических конформных $(p, 1)-$ и $\left(p, p^{\prime}\right)$-моделей, вычисленными в работах [9], [13].

5.2.2. Роль указанных в (5.2) и (5.3) подпредставлений еще предстоит полностью понять с квантово-групповой точки зрения, но они весьма замечательны в контексте соответствия Каждана-Люстига. Возникающие тензорные множители $\mathbb{C}^{n}$ строго коррелировованы с тем фактом, что функции $\psi_{b^{\prime}}(\tau)$ в $(1.1),(1.2)$ даются некоторыми линейными комбинациями характеров, умноженными на многочлены по $\tau$ степени $n-1$.

На центре квантовой группы указанные в (5.2) и (5.3) подпредставления порождаются определенными линейными комбинациями элементов $\widehat{\phi}_{A}$ и $\chi_{A}$ из "базиса Радфорда" и "базиса Дринфельда" соответственно. Для $\overline{\mathcal{U}}_{\mathfrak{q}} s \ell(2)$, например, центральные элементы (4.9) вместе с их $\mathcal{S}$-образами $\frac{p-s}{p} \chi_{s}^{+}-\frac{s}{p} \boldsymbol{\chi}_{p-s}^{-}, 1 \leqslant s \leqslant p-1$, линейно порождают представление $\mathbb{C}^{2} \otimes R_{p-1}$; в логарифмической $(p, 1)$-модели то же представление реализовано на $2(p-1)$ функциях

$$
\tau\left(\frac{p-s}{p} \chi_{s}^{+}(\tau)-\frac{s}{p} \chi_{p-s}^{-}(\tau)\right), \quad \frac{p-s}{p} \chi_{s}^{+}(\tau)-\frac{s}{p} \chi_{p-s}^{-}(\tau),
$$

где $\chi_{s}^{ \pm}(\tau)$ - характеры триплетной алгебры [9]. С другой стороны, $(p+1)$-мерное представление $R_{p+1}$ на центре линейно порождено элементами $\chi_{p}^{ \pm}$и $\chi_{s}^{+}+\chi_{p-s}^{-}$, $1 \leqslant s \leqslant p-1$ (это представление есть идеал, который уже упоминался после (3.2)); в $(p, 1)$-модели то же представление реализовано на линейных комбинациях характеров $\chi_{p}^{ \pm}(\tau), \chi_{s}^{+}(\tau)+\chi_{p-s}^{-}(\tau)$.

Анализ, проведенный для $\mathcal{U}_{p, p^{\prime}}$ в работе [11] (см. Sec. 5.3), дает богатую картину того, как различные следы и псевдоследы, отображенные в центр, комбинируются в подпредставления и того, каким именно образом эти линейные комбинации соответствуют характерам и расширенным характерам из логарифмической $\left(p, p^{\prime}\right)$-модели ${ }^{15)}$. Здесь же мы только отметим, что представление $R_{\text {proj }}$, размерность которого равна $(p+1)\left(p^{\prime}+1\right) / 2$, линейно порождается элементами $\boldsymbol{\chi}_{r, r^{\prime}}^{+}+\boldsymbol{\chi}_{p-r, r^{\prime}}^{-}+\boldsymbol{\chi}_{r, p^{\prime}-r^{\prime}}^{-}+$ $\chi_{p-r, p^{\prime}-r^{\prime}}^{+}\left(\right.$где $\left(r, r^{\prime}\right) \in \mathcal{I}_{1}$ для множества индексов мощности $\left.\left|\mathcal{I}_{1}\right|=(p-1)\left(p^{\prime}-1\right) / 2\right)$, $\chi_{r, p^{\prime}}^{+}+\chi_{p-r, p^{\prime}}^{-}($где $1 \leqslant r \leqslant p-1), \chi_{p, r^{\prime}}^{+}+\chi_{p, p^{\prime}-r^{\prime}}^{-}\left(1 \leqslant r^{\prime} \leqslant p^{\prime}-1\right)$ и $\chi_{p, p^{\prime}}^{ \pm}$. В логарифмической $\left(p, p^{\prime}\right)$-модели то же $S L(2, \mathbb{Z})$-представление реализовано на линейных

${ }^{15)}$ Еще раз: $\mathbb{C}$-линейные комбинации $(3 p-1)\left(3 p^{\prime}-1\right) / 2$ следов и псевдоследов (отображенных в центр) несут те же $S L(2, \mathbb{Z})$-представления, что и определенные $\mathbb{C}[\tau]$-линейные комбинации $2 p p^{\prime}+$ $(p-1)\left(p^{\prime}-1\right) / 2$ характеров $W$-алгебры; полная размерность $(3 p-1)\left(3 p^{\prime}-1\right) / 2$ одна и та же в каждом случае. 
комбинациях следующих характеров $W$-алгебры:

$$
\begin{gathered}
\chi_{r, r^{\prime}}(\tau)+2 \chi_{r, r^{\prime}}^{+}(\tau)+2 \chi_{r, p^{\prime}-r^{\prime}}^{-}(\tau)+2 \chi_{p-r, r^{\prime}}^{-}(\tau)+2 \chi_{p-r, p^{\prime}-r^{\prime}}^{+}(\tau), \quad\left(r, r^{\prime}\right) \in \mathcal{J}_{1}, \\
2 \chi_{p, p^{\prime}-r^{\prime}}^{+}(\tau)+2 \chi_{p, r^{\prime}}^{-}(\tau), \quad 1 \leqslant r^{\prime} \leqslant p^{\prime}-1, \\
2 \chi_{p-r, p^{\prime}}^{+}(\tau)+2 \chi_{r, p^{\prime}}^{-}(\tau), \quad 1 \leqslant r \leqslant p-1, \\
2 \chi_{p, p^{\prime}}^{ \pm}(\tau)
\end{gathered}
$$

(множество индексов $\left(r, r^{\prime}\right)$ также имеет размер $\left.\left|\mathcal{J}_{1}\right|=(p-1)\left(p^{\prime}-1\right) / 2\right)$, где $\chi_{r, r^{\prime}}(\tau)-$ характеры рациональной вирасоровской модели, а $\chi_{r, r^{\prime}}^{ \pm}(\tau)$ - остальные $2 p p^{\prime}$ характеров $W$-алгебры [13]. Приведенные выше комбинации не содержат обобщенных характеров (которые входят туда, где в $S L(2, \mathbb{Z})$-представлении, изоморфном представлению (5.3), появляются множители $\mathbb{C}^{n}$, и которые в действительности и являются источником этих множителей $\mathbb{C}^{n}$ с конформно-полевой точки зрения).

Замечательным свойством $S L(2, \mathbb{Z})$-представления на центре $\mathcal{U}_{p, p^{\prime}}$ является возникновение $R_{\min }$, т.е. $S L(2, \mathbb{Z})$-представления на характерах рациональной вирасоровской модели, несмотря на то что $\mathcal{U}_{p, p^{\prime}}$-представления $\mathcal{X}_{r, r^{\prime}}^{ \pm}$находятся во взаимно однозначном соответствии не со всеми примарными полями $W$-алгебры в логарифмической модели, но с полями за исключением тех, что приходят из рациональной модели.

5.3. За пределами квантовой группы. Две алгебраические структуры на центре квантовой группы наиболее важны с точки зрения соответствия КажданаЛюстига: действие модулярной группы и кольцо Гротендика (последнее является подкольцом в центре, линейно порожденным образами неприводимых представлений при отображении Дринфельда). Возникающие здесь кольца Гротендика, или алгебры Верлинде, неполупросты.

Классификация алгебр Верлинде была предложена в рамках совершенно иного подхода, основанного на двойной аффинной алгебре Гекке (алгебре Чередника) [48], в котором алгебры Верлинде возникают как определенные представления алгебр Чередника; при этом важно, что действие модулярной группы встроено в структуру алгебр Чередника. Таким образом, можно ожидать, что алгебра слияний $(p, 1)$-модели (кольцо Гротендика для $\left.\overline{\mathcal{U}}_{\mathfrak{q}} s \ell(2)\right)$, заданная формулой $(3.1)$, размерности $2 p$, допускает реализацию, связанную с представлением алгебры Чередника. Но поскольку изоморфный образ кольца Гротендика содержится в центре, возникает следующий естественный вопрос: связан ли также весь центр группы $\overline{\mathcal{U}}_{\mathfrak{q}} s \ell(2)$, имеющий размерность $3 p-1$ и наделенный $S L(2, \mathbb{Z})$-действием, с алгеброй Чередника?

B работе [49] было показано, что центр Z алгебры $\overline{\mathcal{U}}_{\mathfrak{q}} s \ell(2)$ как ассоциативная коммутативная алгебра и как $S L(2, \mathbb{Z})$-представление действительно извлекается из пространства представления простейшей алгебры Чередника $\mathcal{H}$, определяемой соотношениями

$$
\begin{gathered}
T X T=X^{-1}, \quad T Y^{-1} T=Y, \\
X Y=\mathfrak{q} Y X T^{2}, \quad(T-\mathfrak{q})\left(T+\mathfrak{q}^{-1}\right)=0
\end{gathered}
$$


для генераторов $T, X, Y$ и их обратных. При этом $P S L(2, \mathbb{Z})$-действие определяется элементами $\tau_{+}=\left(\begin{array}{ll}1 & 1 \\ 0 & 1\end{array}\right)$ и $\tau_{-}=\left(\begin{array}{ll}1 & 0 \\ 1 & 1\end{array}\right)$, реализованными как $\mathcal{H}$-автоморфизмы [48]:

$$
\begin{array}{lll}
\tau_{+}: X \mapsto X, & Y \mapsto \mathfrak{q}^{-1 / 2} X Y, & T \mapsto T, \\
\tau_{-}: X \mapsto \mathfrak{q}^{1 / 2} Y X, & Y \mapsto Y, & T \mapsto T .
\end{array}
$$

Для каждого $p \geqslant 3$ авторы работы [49] построили (6p-4)-мерное (приводимое, но неразложимое) представление алгебры $\mathcal{H}$, в котором подпространство с собственным значением оператора $T$, равным $\mathfrak{q}$ (как и выше, $\mathfrak{q}=e^{i \pi / p}$ ), является $(3 p-1)$-мерным. Структура ассоциативной коммутативной алгебры, индуцированная на этом подпространстве в соответствии с теорией Чередника, совпадает со структурой ассоциативной коммутативной алгебры на центре Z алгебры $\overline{\mathcal{U}}_{\mathfrak{q}} s \ell(2)$. Более того, $S L(2, \mathbb{Z})$-представления, построенные на этом пространстве в рамках работ Чередника и Любашенко, совпадают. Кроме того, образы в центре неприводимых представлений при отображениях Радфорда и Дринфельда можно "поднять" до уровня алгебры $\mathcal{H}$ (как собственные векторы операторов $X+X^{-1}$ и $Y+Y^{-1}$ соответственно) [49].

\section{6. ЗАКЛЮЧЕНИЕ}

Без сомнения, было бы чрезвычайно полезно перевывести такие результаты, как эквивалентность представлений модулярной группы, в более категориальном подходе; это немедленно подсказало бы обобщения. Но “следующая в очереди" квантовая группа после $\overline{\mathcal{U}}_{\mathfrak{q}} s \ell(2)$ и $\mathcal{U}_{p, p^{\prime}}$ - это квантовая $s \ell(2 \mid 1)$ (ср. с замечаниями по ее поводу в [50]), которая уже требует распространения ряда стандартных фактов (например, из работ [21]) на случай квантовых супергрупп.

Центр двойственной по Каждану-Люстигу квантовой группы следует рассматривать как центр соответствующей логарифмической конформной модели; это актуально для применения к граничным состояниям в логарифмических моделях.

\section{ПРИЛОЖКНИЕ}

П.1. Центр и $q$-характеры. Центр Z алгебры Хопфа $U$ можно охарактеризовать как множество

$$
\mathbf{Z}=\left\{y \in U \mid \operatorname{Ad}_{x}(y)=\epsilon(x) y \quad \forall x \in U\right\}
$$

Пространство $q$-характеров $\mathrm{Ch}=\mathrm{Ch}(U) \subset U^{*}$ определяется как

$$
\begin{aligned}
\mathrm{Ch} & =\left\{\beta \in U^{*} \mid \operatorname{Ad}_{x}^{*}(\beta)=\epsilon(x) \beta \quad \forall x \in U\right\}= \\
& =\left\{\beta \in U^{*} \mid \beta(x y)=\beta\left(S^{2}(y) x\right) \quad \forall x, y \in U\right\},
\end{aligned}
$$

где коприсоединенное действие $\operatorname{Ad}_{a}^{*}: U^{*} \rightarrow U^{*}$ имеет вид $\operatorname{Ad}_{a}^{*}(\beta)=\beta\left(S\left(a^{\prime}\right) ? a^{\prime \prime}\right)$, $a \in U, \beta \in U^{*}$. 
П.2. Квазитреугольные алгебры Хопфа. Квазитреугольные алгебры (или косовые (braided) алгебры) Хопфа были введены в [42] (см. также [51]). Квазитреугольная алгебра Хопфа $U$ имеет обратимый элемент $R \in U \otimes U$, удовлетворяющий соотношениям

$$
\begin{aligned}
& \Delta^{\mathrm{op}}(x)=R \Delta(x) R^{-1}, \\
& (\Delta \otimes \mathrm{id})(R)=R_{13} R_{23}, \\
& (\mathrm{id} \otimes \Delta)(R)=R_{13} R_{12},
\end{aligned}
$$

а также уравнению Янга-Бакстера

$$
R_{12} R_{13} R_{23}=R_{23} R_{13} R_{12}
$$

и соотношениям $(\epsilon \otimes \mathrm{id})(R)=1=(\mathrm{id} \otimes \epsilon)(R),(S \otimes S)(R)=R$.

П.3. Квадрат антипода [40]. В любой квазитреугольной алгебре Хопфа квадрат антипода является преобразованием подобия:

$$
S^{2}(x)=\boldsymbol{u} x \boldsymbol{u}^{-1},
$$

где $\boldsymbol{u}-$ канонический элемент,

$$
\boldsymbol{u}=\cdot\left((S \otimes \mathrm{id}) R_{21}\right), \quad \boldsymbol{u}^{-1}=\cdot\left(\left(S^{-1} \otimes S\right) R_{21}\right)
$$

(здесь $\cdot(a \otimes b)=a b)$. Для него выполнено

$$
\Delta(\boldsymbol{u})=M^{-1}(\boldsymbol{u} \otimes \boldsymbol{u})=(\boldsymbol{u} \otimes \boldsymbol{u}) M^{-1},
$$

где, напомним, $M=R_{21} R_{12}$.

П.4. Balancing элемент. Нам требуется также так называемый balancing элемент $\boldsymbol{g} \in U$, который удовлетворяет условиям [40]

$$
S^{2}(x)=\boldsymbol{g} x \boldsymbol{g}^{-1} \quad \forall x \in U, \quad \Delta(\boldsymbol{g})=\boldsymbol{g} \otimes \boldsymbol{g} .
$$

Balancing элемент $\boldsymbol{g}$ позволяет построить "канонический" $q$-характер для любого (неприводимого, поскольку следы нечувствительны к неразложимости) представления $\mathcal{X}$ как (“квантовый”) след:

$$
\operatorname{Tr}_{\mathcal{X}}\left(\boldsymbol{g}^{-1} ?\right) \in \operatorname{Ch}(U)
$$

Для алгебры Хопфа $U$ с правым интегралом $\boldsymbol{\lambda}$ напомним определение комодуля из п. 2.4. Если из комодуля $\boldsymbol{a}$ можно извлечь квадратный корень в $U$, то лемма Дринфельда [40] утверждает, что

$$
g^{2}=a
$$

Добавление при корректуре. Несколько другое применение квантовой группы $\overline{\mathcal{U}}_{\mathfrak{q}} s \ell(2)$ рассматривалось недавно в работе [52]. Дополнительные подробности об устройстве квантовой группы можно также найти в [53]. Функционалы типа следов изучались также в [54]. История изучения квантовой группы $\overline{\mathcal{U}}_{\mathfrak{q}} s \ell(2)$ восходит в действительности к [55]. 
Благодарности. Я признателен М. Финкельбергу, Ю. Фуксу и Ф. Шомерусу за полезные замечания и обсуждения и особенно А. Гайнутдинову за критику. Данная работа частично поддержана РФФИ (грант № 07-01-00523) и Программой поддержки ведущих научных школ (грант № НШ-4401.2006.2).

\section{Список литературы}

[1] L. Alvarez-Gaumé, C. Gomez, G. Sierra, Phys. Lett. B, 220:1-2 (1989), 142-152; Nucl. Phys. B, 319 (1989), 155-186.

[2] G. Moore, N. Reshetikhin, Nucl. Phys. B, 328:3 (1989), 557-574.

[3] V. Pasquier, H. Saleur, Nucl. Phys. B, 330:2-3 (1990), 523-556.

[4] G. Mack, V. Schomerus, Comm. Math. Phys., 134:1 (1990), 139-196.

[5] D. Kazhdan, G. Lusztig, J. Amer. Math. Soc., 6:4 (1993), 905-947; 949-1011; 7:2 (1994), $335-381 ; 383-453$.

[6] M. Finkelberg, Geom. Funct. Anal., 6:2 (1996), 249-267.

[7] V. Gurarie, Nucl. Phys. B, 410:3 (1993), 535-549; arXiv: hep-th/9303160.

[8] M. R. Gaberdiel, H. G. Kausch, Phys. Lett. B, 386:1-4 (1996), 131-137; arXiv: hep-th/9606050.

[9] B. L. Feigin, A. M. Gainutdinov, A. M. Semikhatov, I. Yu. Tipunin, Comm. Math. Phys., 265:1 (2006), 47-93; arXiv: hep-th/0504093.

[10] А. М. Гайнутдинов, А. М. Семихатов, И. Ю. Типунин, Б. Л. Фейгин, ТМФ, 148:3 (2006), 398-427; arXiv: math/0512621.

[11] B. L. Feigin, A. M. Gainutdinov, A. M. Semikhatov, I. Yu. Tipunin, J. Math. Phys., 48:3 (2007), 032303; arXiv: math/0606506.

[12] C. Gomez, M. Ruiz-Altaba, G. Sierra, Quantum Groups in Two-Dimensional Physics, Cambridge University Press, Cambridge, 1996.

[13] B. L. Feigin, A. M. Gainutdinov, A. M. Semikhatov, I. Yu. Tipunin, Nucl. Phys. B, 757:3 (2006), 303-343; arXiv: hep-th/0606196.

[14] B. L. Feigin, A. M. Semikhatov, Nucl. Phys. B, 610:3 (2001), 489-530; arXiv: hep-th/0102078.

[15] B. L. Feigin, A. M. Semikhatov, Nucl. Phys. B, 698:3 (2004), 409-449; arXiv: math/0401164.

[16] G. Felder, Nucl. Phys. B, 317:1 (1989), 215-236.

[17] P. Bouwknegt, J. McCarthy, K. Pilch, Lett. Math. Phys., 23:3 (1991), 193-204; arXiv: hep-th/9108023.

[18] M.A.I. Flohr, Internat. J. Modern Phys. A, 11:22 (1996), 4147-4172; arXiv: hep-th/9509166.

[19] J. Fuchs, S. Hwang, A. M. Semikhatov, I. Yu. Tipunin, Comm. Math. Phys., 247:3 (2004), 713-742; arXiv: hep-th/0306274.

[20] M. Flohr, M. R. Gaberdiel, J. Phys. A, 39:8 (2006), 1955-1967; arXiv: hep-th/0509075.

[21] V. Lyubashenko, Comm. Math. Phys., 172:3 (1995), 467-516; arXiv: hep-th/9405167; "Modular properties of ribbon abelian categories", Proc. 2nd Gauss symposium. Conference A: Mathematics and theoretical physics (Munich, Germany, August 2-7, 1993), Symposia Gaussiana, Berlin-New York, Walter de Gruyter, 1995, 529-579; arXiv: hep-th/9405168; J. Pure Appl. Algebra, 98:3 (1995), 279-327.

[22] V. Lyubashenko, S. Majid, J. Algebra, 166:3 (1994), 506-528.

[23] T. Kerler, Comm. Math. Phys., 168:2 (1995), 353-388; arXiv: hep-th/9402017.

[24] M. R. Gaberdiel, H. G. Kausch, Nucl. Phys. B, 477:1 (1996), 293-318; arXiv: hep-th/9604026.

[25] M.R. Gaberdiel, Internat. J. Modern Phys. A, 18:25 (2003), 4593-4638; arXiv: hep-th/0111260. 
[26] H. Eberle, M. Flohr, J. Phys. A, 39:49 (2006), 15245-15286; arXiv: hep-th/0604097.

[27] M. R. Gaberdiel, H. G. Kausch, Nucl. Phys. B, 538:3 (1999), 631-658; arXiv: hep-th/9807091.

[28] J. Fjelstad, J. Fuchs, S. Hwang, A. M. Semikhatov, I. Yu. Tipunin, Nucl. Phys. B, 633:3 (2002), 379-413; arXiv: hep-th/0201091.

[29] К. Кассель, Квантовые группь, ФАЗИС, М., 1999.

[30] M. E. Sweedler, Hopf Algebras, Benjamin, New York, 1969.

[31] D. E. Radford, J. Algebra, 163:3 (1994), 583-622.

[32] D. E. Radford, J. Algebra, 157:2 (1993), 285-315.

[33] J. Fuchs, "On non-semisimple fusion rules and tensor categories", Contemp. Math., to appear; arXiv: hep-th/0602051.

[34] M. Flohr, H. Knuth, On Verlinde-like formulas in $c_{p, 1}$ logarithmic conformal field theories, arXiv: 0705.0545.

[35] K. Erdmann, E. L. Green, N. Snashall, R. Taillefer, J. Pure Appl. Algebra, 204:2 (2006), 413-454; arXiv: math/0410017.

[36] G. Gotz, T. Quella, V. Schomerus, J. Algebra, 312:2 (2007), 829-848; arXiv: hep-th/0504234.

[37] T. Quella, V. Schomerus, JHEP, 9 (2007), 085; Free fermion resolution of supergroup WZNW models, arXiv: 0706.0744.

[38] H. G. Kausch, Phys. Lett. B, 259:4 (1991), 448-455.

[39] N. Yu. Reshetikhin, M. A. Semenov-Tian-Shansky, J. Geom. Phys., 5:4 (1988), 533-550.

[40] В. Г. Дринфельд, Алгебра и анализ, 1:2 (1989), 30-46.

[41] H.-J. Schneider, Proc. Amer. Math. Soc., 129:7 (2001), 1891-1898.

[42] V. G. Drinfeld, "Quantum groups", Proc. Int. Congr. Math. (Berkeley, 1986), Amer. Math. Soc., Providence, RI, 1987, 798-820.

[43] Ф.Р. Гантмахер, Теория матрии, Наука, М., 1988.

[44] A. M. Gainutdinov, I. Yu. Tipunin, Radford, Drinfeld, and Cardy boundary states in $(1, p)$ logarithmic conformal field models, arXiv: 0711.3430.

[45] N. Yu. Reshetikhin, V. G. Turaev, Comm. Math. Phys., 127:1 (1990), 1-26; Invent. Math., 103:1 (1991), 547-597.

[46] A. Lachowska, J. Algebra, 262:2 (2003), 313-331; arXiv: math/0107098.

[47] R. Bezrukavnikov, A. Lachowska, The small quantum group and the Springer resolution, arXiv: math/0609819.

[48] I. Cherednik, Double Affine Hecke Algebras, London Mathematical Society Lecture Note Series, 319, Cambridge University Press, Cambridge, 2005; Introduction to double Hecke algebras, arXiv: math/0404307.

[49] G. Mutafyan, I. Tipunin, Double affine Hecke algebra in logarithmic conformal field theory, arXiv: 0707.1625.

[50] А. М. Семихатов, ТМФ, 152:3 (2007), 291-346; arXiv: hep-th/0701279.

[51] D. E. Radford, J. Algebra, 151:1 (1992), 1-11.

[52] Y. Arike, Symmetric linear functions of the restricted quantum group $\bar{U}_{q} s_{2}(\mathbb{C})$, arXiv: 0706.1113.

[53] P. Furlan, L. Hadjiivanov, I. Todorov, Zero modes' fusion ring and braid group representations for the extended chiral su(2) WZNW model, arXiv: 0710.1063.

[54] D. E. Radford, S. Westerich, J. Algebra, 301 (2006), 1-34.

[55] А. Ю. Алексеев, Д. В. Глущенков, А. В. Ляховская, Алгебра и анализ, 6:5 (1994), 88-125. 